Andreas Henrici · Thomas Kappeler

\title{
Resonant normal form for even periodic FPU chains
}

Received September 17, 2007

Abstract. We investigate periodic FPU chains with an even number of particles. We show that near the equilibrium point, any such chain admits a resonant Birkhoff normal form of order four which is completely integrable - an important fact which helps explain the numerical experiments of Fermi, Pasta, and Ulam. We analyze the moment map of the integrable approximation of an even FPU chain. Unlike the case of odd FPU chains these integrable systems (generically) exhibit hyperbolic dynamics. As an application we prove that any FPU chain with Dirichlet boundary conditions admits a Birkhoff normal form up to order four and show that a KAM theorem applies.

\section{Introduction}

In this paper we consider FPU chains with an even number $N$ of particles of equal mass, normalized to be one. Such chains have been introduced by Fermi, Pasta, and Ulam [5], as models to test numerically the principle of thermalization as $N$ grows larger and larger. A FPU chain consists of a string of particles moving on the line or the circle interacting only with their nearest neighbors through nonlinear springs. Its Hamiltonian is given by

$$
H_{V}=\frac{1}{2} \sum_{n=1}^{N} p_{n}^{2}+\sum_{n=1}^{N} V\left(q_{n}-q_{n+1}\right),
$$

where $V: \mathbb{R} \rightarrow \mathbb{R}$ is a smooth potential. The corresponding Hamiltonian equations read $(1 \leq n \leq N)$

$$
\dot{q}_{n}=\partial_{p_{n}} H_{V}=p_{n}, \quad \dot{p}_{n}=-\partial_{q_{n}} H_{V}=-V^{\prime}\left(q_{n}-q_{n+1}\right)+V^{\prime}\left(q_{n-1}-q_{n}\right) .
$$

Here $q_{n}$ denotes the displacement of the $n$th particle from its equilibrium position and $p_{n}$ is its momentum. If not stated otherwise, we assume periodic boundary conditions

$$
\left(q_{i+N}, p_{i+N}\right)=\left(q_{i}, p_{i}\right) \quad \forall i \in\{0,1\} .
$$

A. Henrici and T. Kappeler: Institut für Mathematik, Universität Zürich, Winterthurerstrasse 190, CH-8057 Zürich, Switzerland;

e-mail: andreas.henrici@math.uzh.ch, thomas.kappeler@math.uzh.ch

Mathematics Subject Classification (2000): 37J15, 37J35, 37J40, 70H06, 70H08 
Without loss of generality, the potential $V: \mathbb{R} \rightarrow \mathbb{R}$ is assumed to have a Taylor expansion at 0 of the form

$$
V(x)=\kappa\left(\frac{1}{2} x^{2}-\frac{\alpha}{3 !} x^{3}+\frac{\beta}{4 !} x^{4}+\cdots\right),
$$

where $\kappa$ is the (linear) spring constant normalized to be 1 and $\alpha, \beta \in \mathbb{R}$ are parameters measuring the strength of the nonlinear interaction. The minus sign in front of the parameter $\alpha$ in the expansion (3) turns out to be convenient for later computations. Substituting the expression (3) for $V$ into (1) yields the corresponding expansion of $H_{V}$ :

$$
H_{V}=\frac{1}{2} \sum_{n=1}^{N} p_{n}^{2}+\frac{1}{2} \sum_{n=1}^{N}\left(q_{n+1}-q_{n}\right)^{2}+\frac{\alpha}{3 !} \sum_{n=1}^{N}\left(q_{n+1}-q_{n}\right)^{3}+\frac{\beta}{4 !} \sum_{n=1}^{N}\left(q_{n+1}-q_{n}\right)^{4}+\cdots
$$

For any FPU chain, the total momentum $P=N^{-1} \sum_{n=1}^{N} p_{n}$ is an integral of motion, and therefore the center of mass $Q=N^{-1} \sum_{n=1}^{N} q_{n}$ evolves with constant velocity. Hence any FPU chain can be viewed as a family of Hamiltonian systems of $2 N-2$ degrees of freedom, parametrized by the vector of initial conditions $(Q, P) \in \mathbb{R}^{2}$ with Hamiltonian independent of $Q$. In particular, for $N=2$ any FPU chain is integrable, and hence we will concentrate on the case $N \geq 3$. Further, note that for any vector $(Q, P) \in \mathbb{R}^{2}$, the origin in $\mathbb{R}^{N-2}$ is an equilibrium point of the corresponding system. The momentum of such an equilibrium point is given by the constant vector $\left(p_{1}, \ldots, p_{N}\right)=P(1, \ldots, 1)$.

The frequencies $\left(\omega_{k}^{0}\right)_{1 \leq k \leq N-1}$ of the linearization of an arbitrary FPU chain at $(q, p)$ $=(0,0)$ can be easily computed to be

$$
\omega_{k}^{0}=2 \sin \frac{k \pi}{N} .
$$

The corresponding resonance lattice is given by (see Appendix A of [7])

$$
\left\{l=\left(l_{1}, \ldots, l_{N-1}\right) \in \mathbb{Z}^{N-1} \mid \sum_{k=1}^{N-1} l_{k} \sin \frac{k \pi}{N}=0\right\}
$$

and generated by the vectors $l^{(k)}, 1 \leq k \leq N-1$, defined by $l^{(k)}=e_{k}-e_{N-k}$, where $e_{i}$, $1 \leq i \leq N-1$, denotes the standard basis in $\mathbb{R}^{N-1}$.

For any point $(x, y) \in \mathbb{R}^{2 N-2}$ introduce the variables $I=\left(I_{k}\right)_{1 \leq k \leq N-1}$, where

$$
I_{k}=\frac{1}{2}\left(x_{k}^{2}+y_{k}^{2}\right),
$$

as well as $M=\left(M_{k}\right)_{1 \leq k \leq N-1}, J=\left(J_{k}\right)_{1 \leq k \leq N-1}$, and $L=\left(L_{k}\right)_{1 \leq k \leq N-1}$. They are defined on $\mathbb{R}^{2 N-2}$ with values in $\mathbb{R}^{N-1}$ and given by

$$
M_{k}=\frac{1}{2}\left(x_{k} y_{N-k}-x_{N-k} y_{k}\right), J_{k}=\frac{1}{2}\left(x_{k} x_{N-k}+y_{k} y_{N-k}\right), L_{k}=\frac{1}{2}\left(I_{k}-I_{N-k}\right) .
$$

Note that for any $1 \leq k \leq N-1,\left(M_{k}, J_{k}, L_{k}\right)=\left(-M_{N-k}, J_{N-k},-L_{N-k}\right)$ and $I_{k} I_{N-k}=M_{k}^{2}+J_{k}^{2}$, or

$$
\left(\frac{I_{k}+I_{N-k}}{2}\right)^{2}=M_{k}^{2}+J_{k}^{2}+L_{k}^{2}
$$


i.e. $M_{k}, J_{k}, L_{k}$ are the Hopf variables expressed in $x_{k}, y_{k}, x_{N-k}, y_{N-k}$. They describe the image of the Hopf map from the three-dimensional sphere of radius $\frac{1}{2}\left(I_{k}+I_{N-k}\right)$ centered at the orgin of $\mathbb{R}^{4}$. Further, define the function $H_{\alpha, \beta}: \mathbb{R}^{N-1} \rightarrow \mathbb{R}$ by

$$
H_{\alpha, \beta}(I)=2 \sum_{k=1}^{N-1} \sin \frac{k \pi}{N} I_{k}+\frac{1}{4 N} \sum_{k=1}^{N-1} d_{k}^{+} I_{k}^{2}+\frac{\beta-\alpha^{2}}{2 N} \sum_{\substack{l \neq m \\ 1 \leq l, m \leq N-1}} \sin \frac{l \pi}{N} \sin \frac{m \pi}{N} I_{l} I_{m}
$$

where

$$
d_{k}^{+} \equiv d_{k}^{+}(\alpha, \beta):=\alpha^{2}+\left(\beta-\alpha^{2}\right) \sin ^{2} \frac{k \pi}{N}
$$

and let

$$
R_{\alpha, \beta}(J, M):=\frac{\beta-\alpha^{2}}{4 N}\left(R(J, M)+R_{N / 4}(J, M)\right)
$$

where

$$
R(J, M)=4 \sum_{1 \leq k<N / 4} \sin \frac{2 k \pi}{N}\left(J_{k} J_{N / 2-k}-M_{k} M_{N / 2-k}\right)
$$

and

$$
R_{N / 4}(J, M)= \begin{cases}J_{N / 4}^{2}-M_{N / 4}^{2} & \text { if } N / 4 \in \mathbb{N} \\ 0 & \text { otherwise }\end{cases}
$$

Note that for $\alpha, \beta \in \mathbb{R}$ with $\beta=\alpha^{2}$, referred to as the Toda case, $R_{\alpha, \beta}$ vanishes.

In [7] we proved that even FPU chains admit a resonant normal form near the equilibrium point.

Theorem 1.1. If $N \geq 4$ is even, there are canonical coordinates $\left(x_{k}, y_{k}\right)_{1 \leq k \leq N-1}$ such that the Hamiltonian of any FPU chain, when expressed in these coordinates, takes the form $H_{V}^{\text {trunc }}(I, J, M)+O\left(|(x, y)|^{5}\right)$ where

$$
H_{V}^{\text {trunc }}=N P^{2} / 2+H_{\alpha, \beta}(I)-R_{\alpha, \beta}(J, M)
$$

with $\alpha, \beta \in \mathbb{R}$ as in (3) and where $H_{\alpha, \beta}(I)$ and $R_{\alpha, \beta}(J, M)$ are given by $[8$ and $[10]$, respectively.

Note that in the case $\beta \neq \alpha^{2}$, the Hamiltonian $H_{V}$ cannot be transformed into Birkhoff normal form up to order 4 due to resonances. Nevertheless, the Hamiltonian truncated at order $4, H_{V}^{\text {trunc }}$, can be proved to be completely integrable. The form of the resonance lattice introduced above suggests that $I_{k}+I_{N-k}(1 \leq k \leq N / 2)$ are integrals of $H_{V}^{\text {trunc }}$ in involution. To find the remaining commuting integrals we express $H_{\alpha, \beta}(I)$ in terms of $I_{k}+I_{N-k}(1 \leq k \leq N / 2)$ and a remainder term,

$$
H_{\alpha, \beta}(I)=H^{(2)}(I)+H_{\alpha, \beta}^{(4)}(I)+\frac{1}{2 N} \sum_{k=1}^{N / 2-1} d_{k}^{-} I_{k} I_{N-k}
$$


where

$$
\begin{aligned}
H^{(2)}(I)= & 2 \sum_{k=1}^{N / 2-1} \sin \frac{k \pi}{N}\left(I_{k}+I_{N-k}\right)+2 I_{N / 2}, \\
H_{\alpha, \beta}^{(4)}(I)= & \frac{1}{4 N} \sum_{k=1}^{N / 2-1} d_{k}^{+}\left(I_{k}+I_{N-k}\right)^{2}+\frac{\beta}{4 N} I_{N / 2}^{2} \\
& +\frac{\beta-\alpha^{2}}{N} I_{N / 2} \sum_{k=1}^{N / 2-1} \sin \frac{k \pi}{N}\left(I_{k}+I_{N-k}\right) \\
& +\frac{\beta-\alpha^{2}}{2 N} \sum_{\substack{1 \leq k, l<N / 2 \\
k \neq l}} \sin \frac{k \pi}{N} \sin \frac{l \pi}{N}\left(I_{k}+I_{N-k}\right)\left(I_{l}+I_{N-l}\right),
\end{aligned}
$$

and

$$
d_{k}^{-} \equiv d_{k}^{-}(\alpha, \beta):=-\alpha^{2}+\left(\beta-\alpha^{2}\right) \sin ^{2} \frac{k \pi}{N} .
$$

By 77, one has $I_{k} I_{N-k}=J_{k}^{2}+M_{k}^{2}$ for any $1 \leq k \leq N / 2-1$ so that the remainder term $(2 N)^{-1} \sum_{k=1}^{N / 2-1} d_{k}^{-} I_{k} I_{N-k}$ in 14 can be written as

$$
\frac{1}{2 N}\left(\sum_{1 \leq k<N / 4}\left(d_{k}^{-}\left(J_{k}^{2}+M_{k}^{2}\right)+d_{\tilde{k}}^{-}\left(J_{\tilde{k}}^{2}+M_{\tilde{k}}^{2}\right)\right)+d_{N / 4}^{-}\left(J_{N / 4}^{2}+M_{N / 4}^{2}\right)\right),
$$

where the latter term is defined to be 0 if $N / 4 \notin \mathbb{Z}$, and $\tilde{k} \equiv \tilde{k}(k)=N / 2-k$. Combined with the expression 100 for $R_{\alpha, \beta}(J, M)$ the Hamiltonian $H_{V}^{\text {trunc }}$ in $[13)$ then takes the form

$$
H_{V}^{\mathrm{trunc}}=\frac{N P^{2}}{2}+H^{(2)}(I)+H_{\alpha, \beta}^{(4)}(I)+\frac{1}{2 N} \sum_{1 \leq k \leq N / 4} K_{k}(I, J, M)
$$

where for $1 \leq k<N / 4$,

$K_{k}(I, J, M)=d_{k}^{-}\left(J_{k}^{2}+M_{k}^{2}\right)+d_{\tilde{k}}^{-}\left(J_{\tilde{k}}^{2}+M_{\tilde{k}}^{2}\right)-2\left(\beta-\alpha^{2}\right) \sin \frac{2 k \pi}{N}\left(J_{k} J_{\tilde{k}}-M_{k} M_{\tilde{k}}\right)$

and

$$
K_{N / 4}(J, M)= \begin{cases}-\alpha^{2} J_{N / 4}^{2}+\left(\beta-2 \alpha^{2}\right) M_{N / 4}^{2} & \text { if } N \equiv 0 \bmod 4 \\ 0 & \text { otherwise }\end{cases}
$$

Theorem 1.2. Let $N \geq 4$ be an even integer. Then the truncated FPU Hamiltonian $H_{V}^{\text {trunc }}$ given by (13) is completely integrable. If the expansion of $V$ in (3) satisfies $(\alpha, \beta) \neq$ $(0,0)$, then the following $N-1$ quantities are functionally independent integrals in involution:

$$
\left(I_{k}+I_{N-k}\right)_{1 \leq k \leq N / 2}, \quad\left(I_{k}+I_{N / 2+k}\right)_{1 \leq k<N / 4}, \quad\left(K_{k}\right)_{1 \leq k \leq N / 4} .
$$


Remark. In the case $\beta=\alpha^{2}$, the integrals $K_{k}(I, J, M)$ only depend on the action variables $I$, as $M_{j}^{2}+J_{j}^{2}=I_{j} I_{N-j}$ for any $1 \leq j<N / 2$.

In Sections 3 and 4 we present a detailed analysis of the geometry of the moment map of the integrable system of Theorem 1.2. In particular, we show that whenever $\beta \neq \alpha^{2}$, then this integrable system exhibits hyperbolic dynamics.

In Section 5, we will use Theorem 1.1 to show that any FPU chain with Dirichlet boundary conditions admits a Birkhoff normal form up to order 4 by recognizing such a system as an invariant submanifold of a periodic FPU chain. Consider a chain with $N^{\prime}$ $\left(N^{\prime} \geq 3\right.$, not necessarily even) moving particles and Hamiltonian given by

$$
H_{V}^{D}=\frac{1}{2} \sum_{n=1}^{N^{\prime}} p_{n}^{2}+\sum_{n=1}^{N^{\prime}} V\left(q_{n}-q_{n+1}\right) .
$$

Assume that its endpoints are fixed, i.e.

$$
q_{0}=q_{N^{\prime}+1}=0
$$

Theorem 1.3. Any FPU chain with $N^{\prime} \geq 3$ moving particles and Dirichlet boundary conditions admits a Birkhoff normal form of order 4, i.e. there are canonical coordinates $\left(x_{k}, y_{k}\right)_{1 \leq k \leq N^{\prime}}$ such that $H_{V}^{D}$ takes the form

$$
\left(N^{\prime}+1\right) P^{2} / 2+H_{\alpha, \beta}^{D}(I)+O\left(|(x, y)|^{5}\right)
$$

where $I=\left(I_{1}, \ldots, I_{N^{\prime}}\right)$ is given by [5, $\alpha, \beta$ are as in 3 , and $H_{\alpha, \beta}^{D}(I)$ is of the form

$$
\begin{gathered}
2 \sum_{k=1}^{N^{\prime}} s_{k} I_{k}+\frac{1}{16\left(N^{\prime}+1\right)} \sum_{k=1}^{N^{\prime}}\left(\alpha^{2}+3\left(\beta-\alpha^{2}\right) s_{k}^{2}\right) I_{k}^{2} \underbrace{+\frac{\beta-\alpha^{2}}{32\left(N^{\prime}+1\right)} I_{N / 4}^{2}}_{\text {only if } N / 4 \in \mathbb{N}} \\
+\frac{\beta-\alpha^{2}}{16\left(N^{\prime}+1\right)}\left(\sum_{\substack{l \neq m \\
1 \leq l, m \leq N^{\prime}}}^{+N_{l}} 4 s_{l} s_{m} I_{l} I_{m}-\sum_{k=1}^{N^{\prime}} s_{2 k} I_{k} I_{N^{\prime}+1-k}\right) .
\end{gathered}
$$

Note that in 19 , the numbers $s_{k}=\sin \frac{k \pi}{N}=\sin \frac{k \pi}{2 N^{\prime}+2}$ for any $1 \leq k \leq N^{\prime}$ are pairwise different.

Corollary 1.4. Near the equilibrium state, any FPU chain with $N^{\prime}$ moving particles and Dirichlet boundary conditions can be approximated up to order 4 by an integrable system of $N^{\prime}$ harmonic oscillators which are coupled at fourth order except if $\beta=\alpha^{2}$.

Denote by $Q_{\alpha, \beta}^{D}$ the Hessian of $H_{\alpha, \beta}^{D}(I)$ at $I=0$. Note that $Q_{\alpha, \beta}^{D}$ is an $N^{\prime} \times N^{\prime}$-matrix which only depends on the parameters $\alpha$ and $\beta$. 
Theorem 1.5. (i) For any given $\alpha \in \mathbb{R} \backslash\{0\}$, $\operatorname{det}\left(Q_{\alpha, \beta}^{D}\right)$ is a polynomial in $\beta$ of degree $N^{\prime}$ and has $N^{\prime}$ real zeroes (counted with multiplicities). When listed in increasing order, the zeroes $\beta_{k}=\beta_{k}(\alpha)$ satisfy

$$
\beta_{1} \leq \cdots \leq \beta_{\left\lceil\left(N^{\prime}+1\right) / 2\right\rceil}<\alpha^{2}<\beta_{\left\lceil\left(N^{\prime}+3\right) / 2\right\rceil} \leq \cdots \leq \beta_{N^{\prime}}
$$

Moreover, index $\left(Q_{\alpha, \beta}^{D}\right)$, defined as the number of negative eigenvalues of $Q_{\alpha, \beta}^{D}$, is given by

$$
\operatorname{index}\left(Q_{\alpha, \beta}^{D}\right)= \begin{cases}\left\lceil\left(N^{\prime}+1\right) / 2\right\rceil & \text { for } \beta<\beta_{1}, \\ 0 & \text { for } \beta_{\left\lceil\left(N^{\prime}+1\right) / 2\right\rceil}<\beta<\beta_{\left\lceil\left(N^{\prime}+3\right) / 2\right\rceil}, \\ \left\lfloor\left(N^{\prime}-1\right) / 2\right\rfloor & \text { for } \beta>\beta_{N^{\prime}} .\end{cases}
$$

(ii) For $\alpha=0, \operatorname{det}\left(Q_{0, \beta}^{D}\right)$ is a polynomial in $\beta$ of degree $N^{\prime}$, and $\beta=0$ is the only zero of $\operatorname{det}\left(Q_{0, \beta}^{D}\right)$. It has multiplicity $N^{\prime}$, and the index of $Q_{0, \beta}^{D}$ is given by

$$
\operatorname{index}\left(Q_{0, \beta}^{D}\right)= \begin{cases}\left\lceil\left(N^{\prime}+1\right) / 2\right\rceil & \text { for } \beta<0 \\ \left\lfloor\left(N^{\prime}-1\right) / 2\right\rfloor & \text { for } \beta>0\end{cases}
$$

Applications. Theorems 1.3 and 1.5 allow one to apply for any given $\alpha \in \mathbb{R}$ the classical KAM theorem (see e.g. [9]) near the equilibrium point to the FPU chain with Hamiltonian $H_{V}^{D}$ and Dirichlet boundary conditions for a real analytic potential $V(x)=$ $\frac{1}{2} x^{2}-\frac{\alpha}{3 !} x^{3}+\frac{\beta}{4 !} x^{4}+\cdots$ with $\beta \in \mathbb{R} \backslash\left\{\beta_{1}(\alpha), \ldots, \beta_{N^{\prime}}(\alpha)\right\}$. Moreover, as for any given $\alpha \in \mathbb{R} \backslash\{0\}, Q_{\alpha, \beta}^{D}$ is positive definite for $\beta_{\left\lfloor\left(N^{\prime}-1\right) / 2\right\rfloor}(\alpha)<\beta<\beta_{\left\lfloor\left(N^{\prime}+1\right) / 2\right\rfloor}(\alpha)$, one can apply Nekhoroshev's theorem (see e.g. [10]) near the equilibrium point to the FPU chain with Hamiltonian $H_{V}^{D}$ for $V$ with such $\beta$ 's. These perturbation results confirm long standing conjectures-see e.g. [3].

In subsequent work we plan to compare orbits of the FPU chain with Hamiltonian $H_{V}$ with the ones of its integrable approximation $H_{V}^{\text {trunc }}$, with the intention to explain the numerical experiments of Fermi, Pasta, and Ulam.

Related work. Theorems 1.2 and 1.3 improve on earlier results of Rink in [11] and [13], respectively, where the case $\alpha=0$ has been treated. Our approach has been shaped by our work on the Toda lattice [6]. The latter, introduced by Toda [14] and extensively studied, is a special FPU chain which is integrable (i.e. the original Hamiltonian $\mathrm{H}_{V}$-not just $H_{V}^{\text {trunc }}$-is integrable). It turns out that the same canonical transformations which near the equilibrium bring the Toda lattice into Birkhoff normal form can be used for any FPU chain. If $N$ is odd, this transformation brings $H_{V}$ into Birkhoff normal form up to order 4; we have studied this case in detail in [7].

One of the most important open problems in the field of FPU chains is the investigation of the dynamics of these chains when the number of particles gets larger and larger. It is likely that our results on FPU chains with Dirichlet boundary conditions can be used for this purpose. For recent contributions in this direction see e.g. [1], [2]. 


\section{Proof of Theorem 1.2}

Denote by $\{\cdot, \cdot\}$ the standard Poisson bracket on $\mathbb{R}^{2 N-2}$. In a straightforward way one computes the Poisson brackets between the variables $I, M, J, L \in \mathbb{R}^{N-1}$, given by 5 , and (6) (cf. [4, p. 28]):

Lemma 2.1. The Poisson brackets between the variables $I_{k}, J_{k}, M_{k}(1 \leq k \leq N-1)$ are given by

$$
\begin{aligned}
\left\{I_{l}, I_{k}\right\} & =\left\{J_{l}, J_{k}\right\}=\left\{M_{l}, M_{k}\right\}=0, \\
\left\{J_{l}, I_{k}\right\} & =-M_{l}\left(\delta_{k l}-\delta_{k+l, N}\right), \\
\left\{M_{l}, I_{k}\right\} & =J_{l}\left(\delta_{k l}-\delta_{k+l, N}\right),
\end{aligned}
$$

As a consequence, one obtains the following relations between the variables $M_{k}, J_{k}$, and $L_{k}, 1 \leq k \leq N-1$ :

$$
\begin{aligned}
\left\{M_{k}, J_{l}\right\} & =L_{l}\left(\delta_{k+l, N}-\delta_{k l}\right), \\
\left\{J_{k}, L_{l}\right\} & =M_{k}\left(\delta_{k+l, N}-\delta_{k l}\right), \\
\left\{L_{k}, M_{l}\right\} & =J_{l}\left(\delta_{k+l, N}-\delta_{k l}\right) .
\end{aligned}
$$

First note that the list of functions of Theorem 1.2 .

$$
\left(I_{k}+I_{N-k}\right)_{1 \leq k \leq N / 2}, \quad\left(I_{k}+I_{N / 2+k}\right)_{1 \leq k<N / 4}, \quad\left(K_{k}\right)_{1 \leq k \leq N / 4},
$$

contains $N-1$ terms whether $N / 4$ is an integer or not. In addition, for any $1 \leq k<N / 4$, the terms $I_{k}+I_{N-k}, I_{N / 2-k}+I_{N / 2+k}, I_{k}+I_{N / 2+k}, K_{k}$ are functions of the eight variables $x_{k}, y_{k}, x_{N / 2-k}, y_{N / 2-k}, x_{N / 2+k}, y_{N / 2+k}, x_{N-k}$, and $y_{N-k}$, the term $I_{N / 2}$ is a function of the two variables $x_{N / 2}, y_{N / 2}$, and, in the case $N / 4 \in \mathbb{N}$, the terms $I_{N / 4}+I_{3 N / 4}, K_{N / 4}$ are functions of the four variables $x_{N / 4}, y_{N / 4}, x_{3 N / 4}, y_{3 N / 4}$. Hence we obtain a partition of the $2 N-2$ variables $x_{1}, y_{1}, \ldots, x_{N-1}, y_{N-1}$ into $\lfloor N / 4\rfloor+1$ pairwise disjoint sets of variables, and all Poisson brackets between variables of different sets of this partition vanish.

Lemma 2.2. The $N-1$ functions listed in (23) are pairwise in involution.

Proof. The functions in (23) depend on only one of the $\lfloor N / 4\rfloor+1$ pairwise disjoint sets of variables. As the Poisson brackets between terms depending on variables of different sets vanish, it remains to check that functions of (23) with the same $k$ are in involution with each other. In view of the definitions $(16)$ and $(17)$ of $K_{l}$ and $K_{N / 4}$, respectively, and taking into account that $\left(I_{k}\right)_{1 \leq k \leq N-1}$ are pairwise in involution, this amounts to proving that for any $1 \leq l<N / 4$,

$$
\begin{aligned}
\left\{J_{l} J_{N / 2-l}-M_{l} M_{N / 2-l}, I_{l}+I_{N-l}\right\} & =0, \\
\left\{J_{l} J_{N / 2-l}-M_{l} M_{N / 2-l}, I_{N / 2-l}+I_{N / 2+l}\right\} & =0, \\
\left\{J_{l} J_{N / 2-l}-M_{l} M_{N / 2-l}, I_{l}+I_{N / 2+l}\right\} & =0, \\
\left\{J_{N / 4}^{2}-M_{N / 4}^{2}, I_{N / 4}+I_{3 N / 4}\right\} & =0 .
\end{aligned}
$$


First we note that by 21 and 22 one has, for any $1 \leq l<N / 2$,

$$
\begin{aligned}
\left\{J_{l} J_{N / 2-l}-M_{l} M_{N / 2-l}, I_{l}\right\} & =-J_{N / 2-l} M_{l}-M_{N / 2-l} J_{l}, \\
\left\{J_{l} J_{N / 2-l}-M_{l} M_{N / 2-l}, I_{N-l}\right\} & =J_{N / 2-l} M_{l}+M_{N / 2-l} J_{l} .
\end{aligned}
$$

Since the right hand sides of (28) and (29) are invariant under exchanging $l$ and $N / 2-l$, the same must hold for the left hand sides, and we conclude that

$$
\begin{aligned}
& \left\{J_{l} J_{N / 2-l}-M_{l} M_{N / 2-l}, I_{N / 2-l}\right\}=-J_{N / 2-l} M_{l}-M_{N / 2-l} J_{l}, \\
& \left\{J_{l} J_{N / 2-l}-M_{l} M_{N / 2-l}, I_{N / 2+l}\right\}=J_{N / 2-l} M_{l}+M_{N / 2-l} J_{l} .
\end{aligned}
$$

The identities 24 - 26 now follow from the appropriate combinations of 28 - 31). In the same fashion, one concludes that 27 holds.

Proof of Theorem 1.2. In view of Lemma 2.2, it remains to check that the quantities listed in (23) are functionally independent integrals. The independence is easy to verify, and the fact that they are conserved quantities follows from the formula $\sqrt{15}$, , showing that $H_{V}^{\text {trunc }}$ can be written as a function of them.

\section{Foliation by Liouville tori}

In the next two sections we describe the geometry of the moment map of the truncated resonant normal form (13) for any even periodic FPU chain $H_{V}$ with potential $V$ whose expansion $\sqrt{3}$ satisfies $(\alpha, \beta) \neq(0,0)$. The case $\beta=\alpha^{2}$ is special as in this case the normal form 13 is the Birkhoff normal form of order four of the Toda chain. Its foliation is well known-it is the one of uncoupled harmonic oscillators. Hence we will concentrate on the case $\beta \neq \alpha^{2}$ only. The special case $\alpha=0$ has been partially studied by Rink [12]. Surprisingly, it turns out that many of his results continue to hold in the general case. If we use the notation $\tilde{k} \equiv \tilde{k}(k)=N / 2-k$, the integrals of Theorem 1.2 can be grouped as follows:

$$
\left(\mathcal{H}_{k}, \mathcal{H}_{\tilde{k}}, L_{k}, K_{k}\right)_{1 \leq k<N / 4}, \quad I_{N / 2}, \quad \mathcal{H}_{N / 4}, K_{N / 4},
$$

where for $1 \leq k<N / 2$,

$$
\mathcal{H}_{k}:=I_{k}+I_{N-k}, \quad L_{k}:=I_{k}-I_{\tilde{k}}
$$

(Here we used the fact that $L_{k}=\left(I_{k}+I_{N / 2+k}\right)-\left(I_{N / 2-k}+I_{N / 2+k}\right)$ is the difference of two integrals listed in Theorem 1.2 )

Using the assumption $\beta-\alpha^{2} \neq 0$, we rewrite the integrals $\left(K_{l}\right)_{1 \leq l \leq N / 4}$ as follows. Introduce the bifurcation parameter

$$
\gamma \equiv \gamma(\alpha, \beta):=\frac{\alpha^{2}}{\alpha^{2}-\beta}
$$

and note that

$$
d_{k}^{-}=-\alpha^{2}+\left(\beta-\alpha^{2}\right) s_{k}^{2}=\left(\beta-\alpha^{2}\right)\left(\gamma+s_{k}^{2}\right) .
$$


For any $1 \leq k<N / 4$, one has

$$
K_{k}=-d_{k}^{-}\left(J_{k}^{2}+M_{k}^{2}\right)-d_{\tilde{k}}^{-}\left(J_{\tilde{k}}^{2}+M_{\tilde{k}}^{2}\right)+2\left(\beta-\alpha^{2}\right) s_{2 k}\left(J_{k} J_{\tilde{k}}-M_{k} M_{\tilde{k}}\right),
$$

whereas for $k=N / 4$,

$$
K_{k}=\alpha^{2} J_{k}^{2}-\left(\beta-2 \alpha^{2}\right) M_{k}^{2}=\left(\alpha^{2}-\beta\right)\left(\gamma J_{k}^{2}+(1+\gamma) M_{k}^{2}\right) .
$$

Below, we will omit the factor $s_{2 k}\left(\alpha^{2}-\beta\right)$ in $K_{k}$ for simplicity, since it does not influence the geometry of the level sets of the integrals $\left(K_{k}\right)_{1 \leq k \leq N / 4}$.

Each of the $\lfloor N / 4\rfloor+1$ groups of integrals listed in (32) depends only on a subset of the variables $\left\{\left(x_{k}, y_{k}\right)_{1 \leq k \leq N-1}\right\}$. These subsets form a disjoint partition of $\left\{\left(x_{k}, y_{k}\right)_{1 \leq k \leq N-1}\right\}$. More precisely, the following result holds.

Proposition 3.1. The phase space $T^{*} \mathbb{R}^{N-1}$ of the truncated resonant normal form $H_{V}^{\text {trunc }}$ given by (13) is the direct sum of invariant symplectic subspaces,

$$
T^{*} \mathbb{R}^{N-1}=\bigoplus_{0 \leq k \leq N / 4} \mathcal{P}_{k},
$$

where

$$
\mathcal{P}_{k}=\left\{\left(x_{j}, y_{j}\right)_{1 \leq j \leq N-1} \in T^{*} \mathbb{R}^{N-1} \mid x_{j}=y_{j}=0 \forall j \notin\{k, N-k, \tilde{k}, N-\tilde{k}\}\right\} .
$$

The foliation of $T^{*} \mathbb{R}^{N-1}$ by level sets of the integrals 32 is the Cartesian product of the foliations of the $\mathcal{P}_{k}$.

We now analyze the foliations of $\mathcal{P}_{0}, \mathcal{P}_{N / 4}$, and $\mathcal{P}_{k}$ for $0<k<N / 4$ separately. Note that $\mathcal{P}_{0}$ and $\mathcal{P}_{k}(0<k<N / 4)$ can be canonically identified with $T^{*} \mathbb{R}$ and $T^{*} \mathbb{R}^{4}$, respectively, and $\mathcal{P}_{N / 4}$ with $T^{*} \mathbb{R}^{2}$ (if $N / 4 \in \mathbb{N}$ ) or $\{0\}$ (if $N / 4 \notin \mathbb{N}$ ).

Foliation of $\mathcal{P}_{0}$. One easily sees that $I_{N / 2}$ foliates $T^{*} \mathbb{R}$ by circles, centered at the origin.

Foliation of $\mathcal{P}_{N / 4}$ for $N / 4 \in \mathbb{Z}$. Let us study the geometry of the moment map $\mathcal{M}$ : $T^{*} \mathbb{R}^{2} \rightarrow \mathbb{R}^{2}$ defined by the integrable system with commuting integrals $H \equiv H_{N / 4}$ and $K \equiv K_{N / 4}$. It is convenient to introduce the following notation. Denote the standard coordinates of $T^{*} \mathbb{R}^{2}$ by $(x, y)=\left(x_{1}, x_{2}, y_{1}, y_{2}\right)$ and introduce the action variables $I_{j}=$ $\frac{1}{2}\left(x_{j}^{2}+y_{j}^{2}\right)(j=1,2)$, as well as the Hopf variables $M, J, L$ given as in 6 ,

$$
(M, J, L)=\frac{1}{2}\left(x_{1} y_{2}-x_{2} y_{1}, x_{1} x_{2}+y_{1} y_{2}, I_{1}-I_{2}\right) \text {. }
$$

Then the moment map $\mathcal{M}=(H, K)$ takes the form

$$
H=\frac{1}{2}\left(I_{1}+I_{2}\right) \text { and } K=(1+\gamma) M^{2}+\gamma J^{2} .
$$

As already remarked in (7) one has

$$
M^{2}+J^{2}+L^{2}=H^{2} .
$$


Further, we may replace $K$ by $K_{\gamma}$ given by

$$
K_{\gamma}:= \begin{cases}(1+\gamma) M^{2}+\gamma J^{2}, & \gamma \notin\{0,1\} \\ M, & \gamma=0 \\ J, & \gamma=1\end{cases}
$$

First observe that the origin $(x, y)=(0,0)$ of $T^{*} \mathbb{R}^{2}$ is the only critical point of $\mathcal{M}$ with $\operatorname{rank}\left(d_{(x, y)} \mathcal{M}\right)=0$. Moreover,

$$
\mathcal{M}^{-1}\{(0,0)\}=\{(0,0)\} .
$$

The critical points $(x, y) \in T^{*} \mathbb{R}^{2} \backslash\{(0,0)\}$ with $\operatorname{rank}\left(d_{(x, y)} \mathcal{M}\right)=1$ are analyzed by symplectic reduction via the Hamiltonian vector field of $H$. On the sphere $\mathbb{S}_{\rho}^{3}=\{H=$ $\left.\rho^{2} / 4\right\}$ of radius $\rho>0$ in $T^{*} \mathbb{R}^{2}$ define the Hopf map

$$
\mathcal{F}: \mathbb{S}_{\rho}^{3} \rightarrow \mathbb{S}_{r}^{2}, \quad(x, y) \mapsto(M, J, L),
$$

where $r=\left.\sqrt{M^{2}+J^{2}+L^{2}}\right|_{\mathbb{S}_{\rho}^{3}}=\left.H\right|_{\mathbb{S}_{\rho}^{3}}=\rho^{2} / 4$. The fibers of $\mathcal{F}$ are circles obtained by the $\mathbb{S}^{1}$-action of $H$. The reduced system is then given by $\left(\mathbb{S}_{r}^{2}, X_{\gamma}\right)$ where $X_{\gamma}$ denotes the reduced Hamiltonian vector field induced by $K_{\gamma}$. To compute $X_{\gamma}$, note that the equations of motion in the reduced system corresponding to the Hamiltonian $K_{\gamma}$ are given by

$$
\frac{d}{d t}\left(\begin{array}{c}
M \\
J \\
L
\end{array}\right)=\left(\begin{array}{c}
M \\
J \\
L
\end{array}\right) \times\left(\begin{array}{c}
\partial_{M} K_{\gamma} \\
\partial_{J} K_{\gamma} \\
\partial_{L} K_{\gamma}
\end{array}\right)
$$

Indeed, according to the procedure of reduction in Section I.5 of [4], formula (34) follows from

$$
\dot{w}_{j}=\left\{w_{j}, K_{\gamma}\right\}=\sum_{i=1}^{3} \partial_{w_{i}} K_{\gamma}\left\{w_{j}, w_{i}\right\} \quad(1 \leq j \leq 3)
$$

and the commutation relations of the variables $\left(w_{1}, w_{2}, w_{3}\right)=(M, J, L)$ given by Lemma 2.1. We then obtain

$$
X_{\gamma}= \begin{cases}(-2 \gamma J L, 2(1+\gamma) M L,-2 M J), & \gamma \notin\{-1,0\} \\ (0, L,-J), & \gamma=0, \\ (-L, 0, M), & \gamma=-1 .\end{cases}
$$

It turns out that the foliation of $\mathbb{S}_{r}^{2}$ by level sets of $K_{\gamma}$ depends on the bifurcation parameter $\gamma$. If $\gamma=0$, then $( \pm r, 0,0)$ are the only two fixed points of $X_{0}$. They are both elliptic and the level sets of $K_{0}$ in $\mathbb{S}_{r}^{2} \backslash\{( \pm r, 0,0)\}$ are circles. Similarly, for $\gamma=-1,(0, \pm r, 0)$ are the only two fixed points of $X_{-1}$. They are both elliptic and the level sets of $K_{-1}$ in $\mathbb{S}_{r}^{2} \backslash\{(0, \pm r, 0)\}$ are circles. Now let us consider the case $\gamma \in \mathbb{R} \backslash\{0,-1\}$. Then $X_{\gamma}$ admits six fixed points,

$$
( \pm r, 0,0), \quad(0, \pm r, 0), \quad(0,0, \pm r)
$$


two of which are hyperbolic and the remaining four elliptic. Note that the corresponding values of $K_{\gamma}$ are $(1+\gamma) r^{2}, \gamma r^{2}$, and 0 , respectively, and the two hyperbolic fixed points are contained in the same connected component of the inverse image of $K_{\gamma}$ in $\mathbb{S}_{r}^{2}$. This component consists of two great circles where each of the four half circles is a heteroclinic $X_{\gamma}$-orbit connecting the two hyperbolic fixed points.

\begin{tabular}{ccc}
\hline & hyperbolic fixed points & critical value \\
\hline$\gamma<-1$ & $( \pm r, 0,0)$ & $(1+\gamma) r^{2}$ \\
$-1<\gamma<0$ & $(0,0, \pm r)$ & 0 \\
$\gamma>0$ & $(0, \pm r, 0)$ & $\gamma r^{2}$ \\
\hline
\end{tabular}

Let us verify the claimed classification of the two fixed points $(0,0, \varepsilon r)$ with $\varepsilon \in\{ \pm\}$. The other four fixed points are treated in a similar fashion. Near $(0,0, \varepsilon r)$ we choose $M, J$ as coordinates of $\mathbb{S}_{r}^{2}$. The equations of motion induced by $X_{\gamma}$ on $\mathbb{S}_{r}^{2}$ in these coordinates read

$$
\dot{M}=-\varepsilon 2 \gamma J \sqrt{r^{2}-M^{2}-J^{2}}, \quad \dot{J}=\varepsilon 2(1+\gamma) M \sqrt{r^{2}-M^{2}-J^{2}} .
$$

If linearized at $(0,0, \varepsilon r)$, the corresponding linear system is given by the $2 \times 2$-matrix $\varepsilon A$ where

$$
A=2 r\left(\begin{array}{cc}
0 & -\gamma \\
1+\gamma & 0
\end{array}\right)
$$

The eigenvalues of $A$ are given by

$$
\lambda^{2}+4 \gamma(1+\gamma) r^{2}=0, \quad \text { i.e. } \quad \lambda_{1,2}= \pm 2 r \sqrt{-\gamma(1+\gamma)} .
$$

As $-\gamma(1+\gamma)>0$ iff $-1<\gamma<0$ it follows that $\lambda_{1,2}$ are in $\mathbb{R} \backslash\{0\}$ and hence $(0,0, \pm r)$ are both hyperbolic fixed points for $-1<\gamma<0$, whereas they are both elliptic if $\gamma<-1$ or $\gamma>0$. For $-1<\gamma<0$, the inverse image $K_{\gamma}^{-1}(\{0\})$ is given by

$$
\begin{aligned}
K_{\gamma}^{-1}(\{0\}) & =\left\{(M, J, L) \mid(1+\gamma) M^{2}+\gamma J^{2}=0 ; M^{2}+J^{2}+L^{2}=r^{2}\right\} \\
& =\left\{\left.(M, J, L)|M= \pm| \frac{\gamma}{1+\gamma}\right|^{1 / 2} J, M^{2}+J^{2}+L^{2}=r^{2}\right\}
\end{aligned}
$$

whereas for $\gamma<-1$ or $\gamma>0, K_{\gamma}^{-1}(\{0\})=\{(0,0, \pm r)\}$.

Foliation of $\mathcal{P}_{k}$ for $0<k<N / 4$. The foliation of $\mathcal{P}_{k}$ for $0<k<N / 4$ is more complicated than in the case $k=N / 4$. We describe it in detail in the subsequent section.

\section{Integrable system $\left(\mathcal{H}_{k}, \mathcal{H}_{\tilde{k}}, G_{k}, K_{k}\right)$}

In this section we present a detailed study of the geometry of the moment map $\mathcal{M}$ : $T^{*} \mathbb{R}^{4} \rightarrow \mathbb{R}^{4}$ defined by the integrable system $\mathcal{P}_{k}$ with commuting integrals $\mathcal{H}_{k}, \mathcal{H}_{\tilde{k}}, G_{k}$, $K_{k}$ for $1 \leq k<N / 4$. As in Section 3 we restrict to FPU chains with potential $V$ whose expansion (3) satisfies $\beta \neq \alpha^{2}$. We show that in this case the vector field induced by $K_{k}$ 
exhibits hyperbolic dynamics. It is convenient to introduce the following notation. Denote the standard coordinates of $T^{*} \mathbb{R}^{4}$ by $(x, y)$ with $x=\left(x_{i}\right)_{1 \leq i \leq 4}$ and $y=\left(y_{i}\right)_{1 \leq i \leq 4}$, and introduce the action variables $I_{j}=\frac{1}{2}\left(x_{j}^{2}+y_{j}^{2}\right)(1 \leq j \leq 4)$, as well as the Hopf variables $\left(M_{i}, J_{i}, L_{i}\right)_{1 \leq i \leq 2}$ given by

$$
\begin{aligned}
& \left(M_{1}, J_{1}, L_{1}\right)=\frac{1}{2}\left(x_{1} y_{2}-x_{2} y_{1}, x_{1} x_{2}+y_{1} y_{2}, I_{1}-I_{2}\right), \\
& \left(M_{2}, J_{2}, L_{2}\right)=\frac{1}{2}\left(x_{3} y_{4}-x_{4} y_{3}, x_{3} x_{4}+y_{3} y_{4}, I_{3}-I_{4}\right) .
\end{aligned}
$$

By Lemma 2.1 the Poisson brackets between the variables $\left(M_{i}, J_{i}, L_{i}\right)_{1 \leq i \leq 2}$ are given by

$$
\left\{M_{i}, J_{i}\right\}=-L_{i}, \quad\left\{J_{i}, L_{i}\right\}=-M_{i}, \quad\left\{L_{i}, M_{i}\right\}=-J_{i},
$$

whereas all other brackets vanish.

The moment map $\mathcal{M}$ then takes the form

$$
\mathcal{M}: T^{*} \mathbb{R}^{4} \rightarrow \mathbb{R}^{4}, \quad(x, y) \mapsto\left(H_{1}, H_{2}, G, K_{\gamma}\right),
$$

where

$$
H_{1}=\frac{1}{2}\left(I_{1}+I_{2}\right), \quad H_{2}=\frac{1}{2}\left(I_{3}+I_{4}\right), \quad G=L_{1}-L_{2}
$$

and where $K_{\gamma}$ is a scalar multiple of $K_{k}$, given by

$$
K_{\gamma}=\sum_{i=1}^{2} \frac{1}{2} d_{i, \gamma}\left(M_{i}^{2}+J_{i}^{2}\right)+\left(M_{1} M_{2}-J_{1} J_{2}\right)
$$

with the coefficients $d_{1, \gamma}, d_{2, \gamma}$ defined by

$$
d_{1, \gamma}=\frac{\gamma+s_{k}^{2}}{s_{2 k}}, \quad d_{2, \gamma}=\frac{\gamma+c_{k}^{2}}{s_{2 k}}
$$

and $s_{k}=\sin \frac{k \pi}{N}, c_{k}=\cos \frac{k \pi}{N}$. (The definition of the integral $G$ above differs from the one given earlier by a multiple of the integral $H_{1}-H_{2}$ as $I_{1}-I_{3}=L_{1}-L_{2}+H_{1}-H_{2}$.)

First note that the origin $(0,0)$ in $T^{*} \mathbb{R}^{4}$ is the only critical point of $\mathcal{M}$ with $\operatorname{rank}(d \mathcal{N})$ $=0$. Moreover, $\mathcal{M}^{-1}\{(0,0)\}=\{(0,0)\}$. Next observe that upon restriction to $T^{*} \mathbb{R}^{2} \times\{0\}$, one has $G=\frac{1}{2}\left(I_{1}-I_{2}\right)$ and $K_{\gamma}=d_{1, \gamma}\left(H_{1}^{2}-L_{1}^{2}\right)$, hence they are functions of $I_{1}, I_{2}$ alone and $\left.\mathcal{M}\right|_{T^{*} \mathbb{R}^{2} \times\{0\}}$ may be replaced by the map $(x, y) \mapsto\left(I_{1}, I_{2}, 0,0\right)$. The geometry of the latter map is the one of two uncoupled harmonic oscillators. The subspace $\{0\} \times T^{*} \mathbb{R}^{2}$ is treated similarly. It remains to study the restriction of $\mathcal{M}$ to $T^{*} \mathbb{R}^{4} \backslash\left(\left(T^{*} \mathbb{R}^{2} \times\{0\}\right) \cup(\{0\} \times\right.$ $\left.T^{*} \mathbb{R}^{2}\right)$ ). The Hamiltonian vector fields of $H_{1}$ and $H_{2}$ induce a torus action on $T^{*} \mathbb{R}^{2}$. The corresponding symplectic reduction is given by the product of two Hopf maps,

$$
\mathcal{F}: \mathbb{S}_{\rho_{1}}^{3} \times \mathbb{S}_{\rho_{2}}^{3} \rightarrow \mathbb{S}_{r_{1}}^{2} \times \mathbb{S}_{r_{2}}^{2}, \quad(x, y) \mapsto\left(M_{i}, J_{i}, L_{i}\right)_{1 \leq i \leq 2},
$$

where for $i=1,2, \mathbb{S}_{\rho_{i}}^{3}=\left\{H_{i}=\rho_{i}^{2} / 4\right\}$ is a sphere in $T^{*} \mathbb{R}^{2}$ and $r_{i}=\rho_{i}^{2} / 4=$ $\left.\sqrt{M_{i}^{2}+J_{i}^{2}+L_{i}^{2}}\right|_{\mathbb{S}_{\rho_{i}}^{3}}=\left.H_{i}\right|_{\mathbb{S}_{\rho_{i}}^{3}}$. The fibers of $\mathcal{F}$ are 2-dimensional tori, obtained by the $\mathbb{S}^{1} \times \mathbb{S}^{1}$-action of $H_{1} \cdot H_{2}$. The reduced system is then given by $\left(\mathbb{S}_{r_{1}}^{2} \times \mathbb{S}_{r_{2}}^{2}, Y, X{ }_{\gamma}\right)$, where 
$Y$ and $X_{\gamma}$ denote the reduced Hamiltonian vector fields induced by $G$ and $K_{\gamma}$, respectively. To compute $Y$ and $X_{\gamma}$, note that the equations of motion in the reduced system, corresponding to a Hamiltonian $H$, are given by

$$
\frac{d}{d t}\left(\begin{array}{c}
M_{i} \\
J_{i} \\
L_{i}
\end{array}\right)=\left(\begin{array}{c}
M_{i} \\
J_{i} \\
L_{i}
\end{array}\right) \times\left(\begin{array}{c}
\partial_{M_{i}} H \\
\partial_{J_{i}} H \\
\partial_{L_{i}} H
\end{array}\right), \quad i=1,2
$$

- see Section 3 for details. We then obtain

$$
Y=\left(\begin{array}{c}
J_{1} \\
-M_{1} \\
0 \\
-J_{2} \\
M_{2} \\
0
\end{array}\right), \quad X_{\gamma}=\left(\begin{array}{c}
\left(J_{2}-d_{1, \gamma} J_{1}\right) L_{1} \\
\left(d_{1, \gamma} M_{1}+M_{2}\right) L_{1} \\
-\left(M_{1} J_{2}+M_{2} J_{1}\right) \\
\left(J_{1}-d_{2, \gamma} J_{2}\right) L_{2} \\
\left(d_{2, \gamma} M_{2}+M_{1}\right) L_{2} \\
-\left(M_{1} J_{2}+M_{2} J_{1}\right)
\end{array}\right) .
$$

Further, introduce the reduced moment map

$$
\mathcal{M}_{\gamma}: \mathbb{S}_{r_{1}}^{2} \times \mathbb{S}_{r_{2}}^{2} \rightarrow \mathbb{R}^{2}, \quad\left(M_{i}, J_{i}, L_{i}\right)_{1 \leq i \leq 2} \mapsto\left(G, K_{\gamma}\right)
$$

We now study the critical points of $\mathcal{M}_{\gamma}$ with $\operatorname{rank}\left(d \mathcal{M}_{\gamma}\right)=0$, i.e. points of $\mathbb{S}_{r_{1}}^{2} \times \mathbb{S}_{r_{2}}^{2}$ which are fixed points of both $Y$ and $X_{\gamma}$. From the expressions for $Y$ and $X_{\gamma}$ derived above, one easily sees that there are only four such critical points,

$$
\left(M_{i}, J_{i}, L_{i}\right)_{1 \leq i \leq 2}=\varepsilon\left(0,0, r_{1}, 0,0, \pm r_{2}\right),
$$

where $\varepsilon \in\{ \pm\}$. The value of $\mathcal{M}_{\gamma}$ at the critical point $\varepsilon\left(0,0, r_{1}, 0,0,-r_{2}\right)$ is $\left(\varepsilon\left(r_{1}+r_{2}\right), 0\right)$ and

$$
\mathcal{M}_{\gamma}^{-1}\left\{\left(\varepsilon\left(r_{1}+r_{2}\right), 0\right)\right\}=\left\{\varepsilon\left(0,0, r_{1}, 0,0,-r_{2}\right)\right\} .
$$

Computing the Jacobian of the reduced vector field $X_{\gamma}$ at the critical points one sees that they are elliptic fixed points of $X_{\gamma}$. The values of $\mathcal{M}_{\gamma}$ at the other two critical points $\varepsilon\left(0,0, r_{1}, 0,0, r_{2}\right)$ are $\left(\varepsilon\left(r_{1}-r_{2}\right), 0\right)$. The inverse image of $\left(\varepsilon\left(r_{1}-r_{2}\right), 0\right)$ might have several connected components, depending on the values of $\gamma$ and the additional bifurcation parameter

$$
r:=r_{1} / r_{2}>0
$$

Our main results concerning the critical points $\varepsilon\left(0,0, r_{1}, 0,0, r_{2}\right)$ are collected in the following theorem.

Theorem 4.1. Assume that $1 \leq k<N / 4,0<r \leq 1, \varepsilon \in\{ \pm\}$, and $\gamma \in \mathbb{R}$. The critical point $\varepsilon\left(0,0, r_{1}, 0,0, r_{2}\right)$ of $\mathcal{M}_{\gamma}$ is a hyperbolic fixed point of the vector field $X_{\gamma}$ if and only if

$$
\left|\left(\gamma+s_{k}^{2}\right) \sqrt{r}+\left(\gamma+c_{k}^{2}\right) \frac{1}{\sqrt{r}}\right|<2 s_{2 k} .
$$

Otherwise it is an elliptic fixed point of $X_{\gamma}$. If $(38)$ is satisfied, the stable and unstable manifolds of $\varepsilon\left(0,0, r_{1}, 0,0, r_{2}\right)$ both have dimension two. In the case $r<1$, the connected component of $\mathcal{M}_{\gamma}^{-1}\left\{\varepsilon\left(r_{1}-r_{2}, 0\right)\right\}$ containing $\varepsilon\left(0,0, r_{1}, 0,0, r_{2}\right)$ is a 2-dimensional torus 


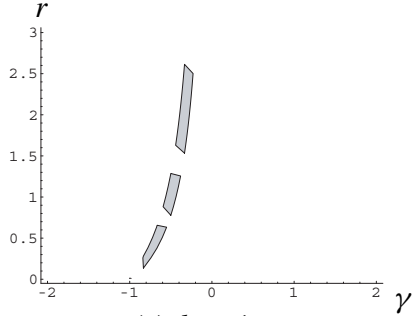

(a) $k=1$

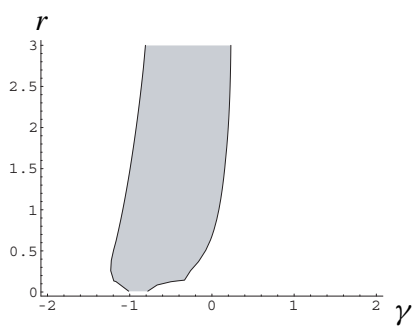

(c) $k=10$

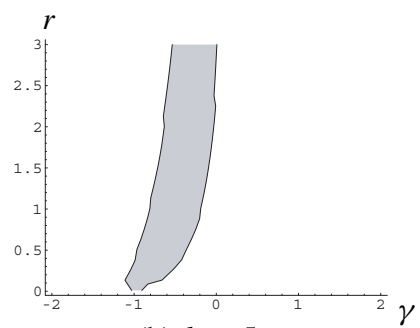

(b) $k=5$

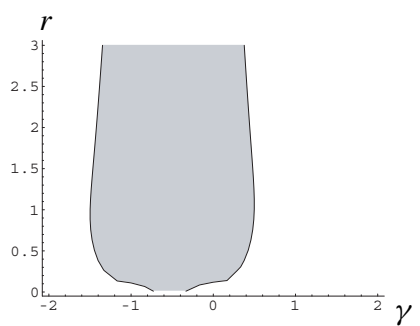

(d) $k=24$

Fig. 1. Subsets of parameters $(\gamma, r)$ with hyperbolic dynamics of $X_{\gamma}(N=100)$.

pinched at $\varepsilon\left(0,0, r_{1}, 0,0, r_{2}\right)$ and consists of homoclinic $X_{\gamma}$-orbits. In the case $r=1$, $\mathcal{M}_{\gamma}^{-1}\{(0,0)\}$ is a 2-dimensional torus pinched at the two points $\pm\left(0,0, r_{1}, 0,0, r_{1}\right)$, and $\mathcal{M}_{\gamma}^{-1}\{(0,0)\} \backslash\left\{ \pm\left(0,0, r_{1}, 0,0, r_{1}\right)\right\}$ consists of heteroclinic $X_{\gamma}$-orbits.

To prove Theorem 4.1 we separately treat for any given $1 \leq k<N / 4$ three subsets of the domain of the parameters $\gamma$ and $r$. The results for these three cases are stated in detail in Propositions 4.2 4.4 below.

Note that the inverse image $\mathcal{M}_{\gamma}^{-1}\left\{\left(\varepsilon\left(r_{1}-r_{2}\right), 0\right)\right\}$ is invariant under the action of the vector field $Y$. The orbits of this action can be easily described,

$$
\left\{\left(R_{-\phi}\left(M_{1}, J_{1}\right), L_{1}, R_{\phi}\left(M_{2}, J_{2}\right), L_{2}\right)|| \phi \mid \leq \pi\right\},
$$

where $R_{\phi}(u, v)$ denotes the image of $(u, v) \in \mathbb{R}^{2}$ under the counterclockwise rotation $R_{\phi}$ by the angle $\phi$. Hence given $L_{1}$ and $L_{2}$ with $\left|L_{i}\right|<r_{i}$ for $i=1,2$ there exists a unique point $\left(\hat{M}_{i}, \hat{J}_{i}, L_{i}\right)_{1 \leq i \leq 2}$ on such an orbit with the property that

$$
\left(\hat{M}_{1}, \hat{J}_{1}\right)=\left(\sqrt{r_{1}^{2}-L_{1}^{2}}, 0\right) \quad \text { and } \quad\left(\hat{M}_{2}, \hat{J}_{2}\right)=\sqrt{r_{2}^{2}-L_{2}^{2}}(\cos \alpha, \sin \alpha)
$$

for some $0 \leq \alpha<2 \pi$. We denote the corresponding $Y$-orbit by $\mathcal{L}\left(L_{1}, L_{2}, \alpha\right)$, i.e.

$$
\mathcal{L}\left(L_{1}, L_{2}, \alpha\right)=\left\{\left(R_{-\phi}\left(\sqrt{r_{1}^{2}-L_{1}^{2}}, 0\right), L_{1}, R_{\alpha+\phi}\left(\sqrt{r_{2}^{2}-L_{2}^{2}}, 0\right), L_{2}\right)|| \phi \mid \leq \pi\right\} .
$$

As $G$ and $K_{\gamma}$ commute, $K_{\gamma}$ is invariant along any orbit of the vector field $Y$ and we conclude that

$$
K_{\gamma}\left(\left(M_{i}, J_{i}, L_{i}\right)_{1 \leq i \leq 2}\right)=\frac{1}{2} \sum_{i=1}^{2} d_{i, \gamma}\left(r_{i}^{2}-L_{i}^{2}\right)+\sqrt{\left(r_{1}^{2}-L_{1}^{2}\right)\left(r_{2}^{2}-L_{2}^{2}\right)} \cos \alpha .
$$


Let us now determine $\mathcal{M}_{\gamma}^{-1}\left\{\left(\varepsilon\left(r_{1}-r_{2}\right), 0\right)\right\}$ for $\varepsilon=1$ and $r \leq 1$. (The case where $r>1$ and/or $\varepsilon=-1$ is treated in a similar fashion.) Let $\left(M_{i}, J_{i}, L_{i}\right)_{1 \leq i \leq 2}$ be an element of $\mathcal{M}_{\gamma}^{-1}\left\{\left(r_{1}-r_{2}, 0\right)\right\} \backslash\left\{\left(0,0, r_{1}, 0,0, r_{2}\right)\right\}$. Then $r_{2}-L_{2}=r_{1}-L_{1}$ and $K_{\gamma}\left(\left(M_{i}, J_{i}, L_{i}\right)_{1 \leq i \leq 2}\right)$ $=0$. First note that $L_{1}<r_{1}$. Indeed, if $L_{1}=r_{1}$, then $L_{2}=r_{2}$ and $\left(M_{i}, J_{i}\right)=(0,0)$ for $i=1,2$, contradicting our assumption on the point considered. Hence in the expression for $K_{\gamma}$ displayed above we can factor out $r_{1}-L_{1}$ and the equation $K_{\gamma}=0$ reads

$$
0=d_{1, \gamma}\left(r_{1}+L_{1}\right)+d_{2, \gamma}\left(r_{2}+L_{2}\right)+2 \sqrt{\left(r_{1}+L_{1}\right)\left(r_{2}+L_{2}\right)} \cos \alpha .
$$

Next let us consider the case where $L_{1}=-r_{1}$. Then $\left(M_{1}, J_{1}, L_{1}\right)=-\left(0,0, r_{1}\right)$ and 42 ) reads $\left(\gamma+c_{k}^{2}\right)\left(r_{2}+L_{2}\right)=0$. Hence either $L_{2}=-r_{2}$ or $\gamma=-c_{k}^{2}$. In the case $L_{2}=-r_{2}$ it follows from $r_{2}-L_{2}=r_{1}-L_{1}=2 r_{1}$ that $r_{2}=r_{1}$ and $L_{2}=L_{1}$. As a consequence, $\left(M_{2}, J_{2}, L_{2}\right)=-\left(0,0, r_{1}\right)$. On the other hand, if $\gamma=-c_{k}^{2}$ and $r_{1}<r_{2}$, then

$$
-r_{2}<r_{2}-2 r_{1}=L_{2}<r_{2} \quad \text { and } \quad M_{2}^{2}+J_{2}^{2}=r_{2}^{2}-L_{2}^{2}=4 r_{1}\left(r_{2}-r_{1}\right) \text {. }
$$

If $L_{1} \neq-r_{1}$ (and hence $L_{2} \neq-r_{2}$ as $\left.r_{1} \leq r_{2}\right)$ we set, for $-r_{1}<L_{1}<r_{1}$,

$$
Q\left(L_{1}\right):= \begin{cases}\sqrt{\frac{r_{1}+L_{1}}{2 r_{2}+L_{1}-r_{1}}} & \text { if } r_{1}<r_{2}, \\ 1 & \text { if } r_{1}=r_{2} .\end{cases}
$$

Then $0<Q<\sqrt{r}$ and for $r<1, Q$ is increasing on $-r_{1}<L_{1}<r_{1}$. After division by $\frac{1}{s_{2 k}} \sqrt{\left(r_{1}+L_{1}\right)\left(r_{2}+L_{2}\right)}$ equation 42 reads

$$
\left(\gamma+s_{k}^{2}\right) Q+\left(\gamma+c_{k}^{2}\right) \frac{1}{Q}+2 s_{2 k} \cos \alpha=0 .
$$

To investigate the solutions of 44 we distinguish between three cases: $r=1,\left[\gamma+c_{k}^{2}=0\right.$ and $r<1]$, and $\left[\gamma+c_{k}^{2} \neq 0\right.$ and $\left.r<1\right]$.

Let us first treat the case $r=1$. Then $Q \equiv 1$ and equation (44) takes the form

$$
2 \gamma+1=-2 s_{2 k} \cos \alpha
$$

which is independent of $L_{1}$.

Proposition 4.2. Let $\gamma \in \mathbb{R}$ be arbitrary and assume $1 \leq k<N / 4$ and $r=1$. Then the following statements hold:

(i) If $|2 \gamma+1|>2 s_{2 k}$, then

$$
\mathcal{M}_{\gamma}^{-1}\{(0,0)\}=\left\{\varepsilon\left(0,0, r_{1}, 0,0, r_{1}\right) \mid \varepsilon= \pm\right\}
$$

and $\pm\left(0,0, r_{1}, 0,0, r_{1}\right)$ are both elliptic fixed points of the vector field $X_{\gamma}$. 
(ii) If $|2 \gamma+1|<2 s_{2 k}$, then $\mathcal{M}_{\gamma}^{-1}\{(0,0)\}=\mathcal{N}_{\alpha} \cup \mathcal{N}_{-\alpha}$, where $\alpha$ is the unique angle satisfying $0<\alpha<\pi$ and $2 \gamma+1=-2 s_{2 k} \cos \alpha$, and where for any $-\pi \leq \beta \leq \pi$,

$$
\mathcal{N}_{\beta}=\bigcup_{\substack{\left|L_{1}\right| \leq r_{1} \\ L_{2}=L_{1}}} \mathcal{L}\left(L_{1}, L_{2}, \beta\right)
$$

with $\mathcal{L}\left(L_{1}, L_{2}, \beta\right)$ given by $(40)$. Both points, $\pm\left(0,0, r_{1}, 0,0, r_{1}\right)$, are hyperbolic fixed points of $X_{\gamma}$, and their stable and unstable manifolds each have dimension two. The set $\mathcal{N}_{\alpha} \backslash\left\{ \pm\left(0,0, r_{1}, 0,0, r_{1}\right)\right\}$ consists of heteroclinic $X_{\gamma}$-orbits from $\left(0,0, r_{1}, 0,0, r_{1}\right)$ to $-\left(0,0, r_{1}, 0,0, r_{1}\right)$, whereas $\mathcal{N}_{-\alpha} \backslash\left\{ \pm\left(0,0, r_{1}, 0,0, r_{1}\right)\right\}$ consists of heteroclinic $X_{\gamma}$-orbits with opposite direction. Topologically, $\mathcal{M}_{\gamma}^{-1}\{(0,0)\}$ is a 2-dimensional torus, pinched at each of the two fixed points $\pm\left(0,0, r_{1}, 0,0, r_{1}\right)$.

(iii) If $2 \gamma+1=-2 s_{2 k}$, then $\alpha=0$ and $\mathcal{M}_{\gamma}^{-1}\{(0,0)\}=\mathcal{N}_{0}$, whereas if $2 \gamma+1=2 s_{2 k}$, then $\alpha=\pi$ and $\mathcal{M}_{\gamma}^{-1}\{(0,0)\}=\mathcal{N}_{\pi}$. In both cases, $\pm\left(0,0, r_{1}, 0,0, r_{1}\right)$ are elliptic fixed points of $X_{\gamma}$. On $\mathcal{N}_{0} \cup \mathcal{N}_{\pi}$, any $X_{\gamma}$-orbit is periodic and coincides with the corresponding $Y$-orbit at least up to orientation.

Proof. (i) By a straightforward computation one shows that under the given assumptions, both points $\pm\left(0,0, r_{1}, 0,0, r_{1}\right)$ are elliptic fixed points of $X_{\gamma}$. In view of equation (45), item (i) then easily follows.

(ii) By the discussion preceding Proposition 4.2 it follows that the inverse image $\mathcal{M}_{\gamma}^{-1}\{(0,0)\}$ is as claimed. Again by a straightforward computation one shows that both fixed points $\pm\left(0,0, r_{1}, 0,0, r_{1}\right)$ are hyperbolic. To see that $\mathcal{N}_{\alpha} \backslash\left\{ \pm\left(0,0, r_{1}, 0,0, r_{1}\right)\right\}$ consists of heteroclinic orbits of the vector field $X_{\gamma}$, consider the third component $\left(X_{\gamma}\right)_{3}$ of $X_{\gamma}$ (cf. (37)). Any element $\left(M_{i}, J_{i}, L_{i}\right)_{1 \leq i \leq 2}$ in $\mathcal{N}_{\alpha}$ is of the form

$\left(M_{1}, J_{1}\right)=\sqrt{r_{1}^{2}-L_{1}^{2}}(\cos \phi,-\sin \phi), \quad\left(M_{2}, J_{2}\right)=\sqrt{r_{1}^{2}-L_{1}^{2}}(\cos (\alpha+\phi), \sin (\alpha+\phi))$.

Thus

$\left(X_{\gamma}\right)_{3}=-\left(M_{1} J_{2}+M_{2} J_{1}\right)=-\left(r_{1}^{2}-L_{1}^{2}\right)(\cos \phi \sin (\alpha+\phi)-\cos (\alpha+\phi) \sin \phi)$.

Hence $\left(X_{\gamma}\right)_{3}=-\left(r_{1}^{2}-L_{1}^{2}\right) \sin \alpha<0$ for any point in $\mathcal{N}_{\alpha} \backslash\left\{ \pm\left(0,0, r_{1}, 0,0, r_{1}\right)\right\}$. As the last component of $X_{\gamma}$ coincides with the third one, it follows that any $X_{\gamma}$-orbit on $\mathcal{N}_{\alpha} \backslash$ $\left\{ \pm\left(0,0, r_{1}, 0,0, r_{1}\right)\right\}$ originates from $\left(0,0, r_{1}, 0,0, r_{1}\right)$ and ends at $-\left(0,0, r_{1}, 0,0, r_{1}\right)$. The orbits on $\mathcal{N}_{-\alpha}$ are analyzed in a similar way.

(iii) Clearly, if $2 \gamma+1=-2 s_{2 k}$, one has $\mathcal{M}_{\gamma}^{-1}\{(0,0)\}=\mathcal{N}_{0}$ and one verifies in a straightforward way that $\pm\left(0,0, r_{1}, 0,0, r_{1}\right)$ are elliptic fixed points of $X_{\gamma}$. According to (47), $\left(X_{\gamma}\right)_{3}$ vanishes identically on $\mathcal{N}_{0}$. Further, $2 \gamma+1=-2 s_{2 k}$ implies that $1+d_{2, \gamma}=$ $-1-d_{1, \gamma}$. In view of 46 it then follows that

$$
X_{\gamma}=\left(1+d_{2, \gamma}\right) L_{2} \cdot Y
$$

The claimed statements for the case $2 \gamma+1=2 s_{2 k}$ are proved in a similar fashion. 
Next we consider the case where $r<1$ and $\gamma+c_{k}^{2}=0$. Then $\gamma+s_{k}^{2}=-c_{2 k}$ and hence

$$
d_{1, \gamma}=-c_{2 k} / s_{2 k} \quad \text { and } \quad d_{2, \gamma}=0
$$

Thus equation (44) takes the form

$$
c_{2 k} Q\left(L_{1}\right)=2 s_{2 k} \cos \alpha
$$

Note that $0<c_{2 k}<1$ as $1 \leq k<N / 4$.

Proposition 4.3. Assume that $1 \leq k<N / 4,0<r<1$, and $\gamma+c_{k}^{2}=0$. Then the following statements hold:

(i) If $\sqrt{r}>2 s_{2 k} / c_{2 k}$, then the connected component of $\mathcal{M}_{\gamma}^{-1}\left\{\left(r_{1}-r_{2}, 0\right)\right\}$ containing the critical point $\left(0,0, r_{1}, 0,0, r_{2}\right)$ consists of this point alone. It is an elliptic fixed point of $X_{\gamma}$.

(ii) If $\sqrt{r} \leq 2 s_{2 k} / c_{2 k}$, then

$$
\mathcal{M}_{\gamma}^{-1}\left\{\left(r_{1}-r_{2}, 0\right)\right\}=\bigcup_{\substack{\left|L_{1}\right| \leq r_{1} \\ L_{2}=L_{1}+r_{2}-r_{1}}} \mathcal{L}\left(L_{1}, L_{2}, \alpha_{L_{1}}\right) \cup \mathcal{L}\left(L_{1}, L_{2},-\alpha_{L_{1}}\right)
$$

where for any $\left|L_{1}\right| \leq r_{1}, \alpha_{L_{1}}$ is the unique angle satisfying

$$
c_{2 k} Q\left(L_{1}\right)=2 s_{2 k} \cos \alpha_{L_{1}} \quad \text { and } \quad 0 \leq \alpha_{L_{1}} \leq \pi / 2
$$

and $Q\left(L_{1}\right)$ denotes the function defined by (43), continuously extended to the closed interval $\left[-L_{1}, L_{1}\right]$. Furthermore, the connected component of $\mathcal{M}_{\gamma}^{-1}\left\{\left(r_{1}-r_{2}, 0\right)\right\}$ containing $\left(0,0, r_{1}, 0,0, r_{2}\right)$ consists of homoclinic $X_{\gamma}$-orbits which originate and end at $\left(0,0, r_{1}, 0,0, r_{2}\right)$. Topologically, it is a 2-dimensional torus, pinched at $\left(0,0, r_{1}, 0,0, r_{2}\right)$.

If $\sqrt{r}<2 s_{2 k} / c_{2 k}$, then $\left(0,0, r_{1}, 0,0, r_{2}\right)$ is a hyperbolic fixed point of $X_{\gamma}$ and its stable and unstable manifolds each have dimension two. If $\sqrt{r}=2 s_{2 k} / c_{2 k}$, $\left(0,0, r_{1}, 0,0, r_{2}\right)$ is an elliptic fixed point of $X_{\gamma}$.

Proof. (i) By a straightforward computation one shows that under the given assumptions, $\left(0,0, r_{1}, 0,0, r_{2}\right)$ is an elliptic fixed point of $X_{\gamma}$. In view of 48$)$ and the discussion of the case $L_{1}= \pm r_{1}$ item (i) then follows easily.

(ii) Again by the discussion preceding Proposition 4.2 it follows that $\mathcal{M}_{\gamma}^{-1}\left\{\left(r_{1}-r_{2}, 0\right)\right\}$ is as claimed. Again by a straightforward computation one sees that $\left(0,0, r_{1}, 0,0, r_{2}\right)$ is a hyperbolic fixed point of $X_{\gamma}$ if $\sqrt{r}<2 s_{2 k} / c_{2 k}$, and an elliptic one if $\sqrt{r}=2 s_{2 k} / c_{2 k}$. Next consider a point $\left(M_{i}, J_{i}, L_{i}\right)_{1 \leq i \leq 2}$ in $\mathcal{M}_{\gamma}^{-1}\left\{\left(r_{1}-r_{2}, 0\right)\right\}$ with

$$
\begin{aligned}
& \left(M_{1}, J_{1}\right)=\sqrt{r_{1}^{2}-L_{1}^{2}}(\cos \phi,-\sin \phi), \\
& \left(M_{2}, J_{2}\right)=\sqrt{r_{2}^{2}-L_{2}^{2}}\left(\cos \left(\alpha_{L_{1}}+\phi\right), \sin \left(\alpha_{L_{1}}+\phi\right)\right)
\end{aligned}
$$


where $\left|L_{1}\right|<r_{1}$. Then the third component of $X_{\gamma}$ is given by (cf. (47))

$$
\left(X_{\gamma}\right)_{3}=-\sqrt{r_{1}^{2}-L_{1}^{2}} \sqrt{r_{2}^{2}-L_{2}^{2}} \sin \alpha_{L_{1}} \text {. }
$$

Hence $\left(X_{\gamma}\right)_{3}=\left(X_{\gamma}\right)_{6}<0$. It follows that the $X_{\gamma}$-orbit passing through such a point originates at $\left(0,0, r_{1}, 0,0, r_{2}\right)$ and then reaches a point of the form $\left(0,0,-r_{1}, M_{2}, J_{2}, L_{2}\right)$ with

$$
L_{2}=r_{2}-2 r_{1}>-r_{2} \quad \text { and } \quad\left(M_{2}, J_{2}\right)=\sqrt{r_{2}^{2}-L_{2}^{2}}(\cos (\pi+\tilde{\phi}), \sin (\pi+\tilde{\phi})) .
$$

At this point the vector field $X_{\gamma}$ is given by

$$
\left(-r_{1} J_{2},-r_{1} M_{2}, 0,0,0,0\right) \text {. }
$$

Note that this vector does not vanish as $M_{2}^{2}+J_{2}^{2}=r_{2}^{2}-L_{2}^{2}>0$. Similarly, at a point $\left(M_{i}, J_{i}, L_{i}\right)_{1 \leq i \leq 2}$ in $\mathcal{M}_{\gamma}^{-1}\left\{\left(r_{1}-r_{2}, 0\right)\right\}$ satisfying

$$
\begin{aligned}
& \left(M_{1}, J_{1}\right)=\sqrt{r_{1}^{2}-L_{1}^{2}}(\cos \phi,-\sin \phi), \\
& \left(M_{2}, J_{2}\right)=\sqrt{r_{2}^{2}-L_{2}^{2}}\left(\cos \left(-\alpha_{L_{1}}+\phi\right), \sin \left(-\alpha_{L_{1}}+\phi\right)\right)
\end{aligned}
$$

and $\left|L_{1}\right|<r_{1}$ one has

$$
\left(X_{\gamma}\right)_{3}=\sqrt{r_{1}^{2}-L_{1}^{2}} \sqrt{r_{2}^{2}-L_{2}^{2}} \sin \alpha_{L_{1}} .
$$

Hence $\left(X_{\gamma}\right)_{3}=\left(X_{\gamma}\right)_{6}>0$. It follows that the $X_{\gamma}$-orbit passing through such a point ends up at $\left(0,0, r_{1}, 0,0, r_{2}\right)$ and passes through a point of the form $\left(0,0,-r_{1}, M_{2}, J_{2}, L_{2}\right)$ with $\left(M_{2}, J_{2}, L_{2}\right)$ as in 49 . We conclude that the connected component of $\mathcal{M}_{\gamma}^{-1}\left\{\left(r_{1}-r_{2}, 0\right)\right\}$ containing $\left(0,0, r_{1}, 0,0, r_{2}\right)$ consists of homoclinic $X_{\gamma}$-orbits originating and ending at $\left(0,0, r_{1}, 0,0, r_{2}\right)$.

Finally, let us treat the case $r<1$ and $\gamma+c_{k}^{2} \neq 0$. Denote by $Q\left(L_{1}\right)$ the function defined by 43 , extended continuously to the closed interval $\left[-r_{1}, r_{1}\right]$. Further, introduce the function

$$
f:(0, \sqrt{r}) \rightarrow \mathbb{R}, \quad q \mapsto\left(\gamma+s_{k}^{2}\right) q+\left(\gamma+c_{k}^{2}\right) \frac{1}{q}
$$

Note that $\lim _{q \searrow 0}|f(q)|=\infty$.

Proposition 4.4. Assume that $1 \leq k<N / 4,0<r<1$, and $\gamma+c_{k}^{2} \neq 0$. Then the following statements hold:

(i) If $|f(\sqrt{r})| \geq 2 s_{2 k}$, then the connected component of $\mathcal{M}_{\gamma}^{-1}\left\{\left(r_{1}-r_{2}, 0\right)\right\}$ containing the critical point $\left(0,0, r_{1}, 0,0, r_{2}\right)$ consists of this point alone. It is an elliptic fixed point of $X_{\gamma}$. 
(ii) If $|f(\sqrt{r})|<2 s_{2 k}$, then there exists $-r_{1}<l_{\gamma, r}<r_{1}$ such that the connected component of $\mathcal{M}_{\gamma}^{-1}\left\{\left(r_{1}-r_{2}, 0\right)\right\}$ containing $\left(0,0, r_{1}, 0,0, r_{2}\right)$ is given by

$$
\bigcup_{\substack{l_{\gamma, r} \leq L_{1} \leq r_{1} \\ L_{2}=L_{1}+r_{2}-r_{1}}} \mathcal{L}\left(L_{1}, L_{2}, \alpha_{L_{1}}\right) \cup \mathcal{L}\left(L_{1}, L_{2},-\alpha_{L_{1}}\right)
$$

where for any $l_{\gamma, r} \leq L_{1} \leq r_{1}, \alpha_{L_{1}}$ is the unique angle satisfying $0 \leq \alpha_{L_{1}} \leq \pi$ and

$$
f\left(Q\left(L_{1}\right)\right)=-2 s_{2 k} \cos \alpha_{L_{1}} .
$$

The point $\left(0,0, r_{1}, 0,0, r_{2}\right)$ is a hyperbolic fixed point of $X_{\gamma}$ and its stable and unstable manifolds each have dimension two. The connected component of the set $\mathcal{M}_{\gamma}^{-1}\left\{\left(r_{1}-r_{2}, 0\right)\right\}$ containing $\left(0,0, r_{1}, 0,0, r_{2}\right)$ consists of homoclinic $X_{\gamma}$-orbits which originate and end at $\left(0,0, r_{1}, 0,0, r_{2}\right)$. Topologically, it is a 2-dimensional torus, pinched at $\left(0,0, r_{1}, 0,0, r_{2}\right)$.

Proof. (i) By a straightforward computation one shows that under the given assumptions, $\left(0,0, r_{1}, 0,0, r_{2}\right)$ is a (possibly degenerate) elliptic fixed point of $X_{\gamma}$. We have already seen that under the given assumption, $\mathcal{M}_{\gamma}^{-1}\left\{\left(r_{1}-r_{2}, 0\right)\right\} \backslash\left\{\left(0,0, r_{1}, 0,0, r_{2}\right)\right\}$ is the set of points $\left(M_{i}, J_{i}, L_{i}\right)_{1 \leq i \leq 2}$ satisfying $L_{2}=L_{1}+r_{2}-r_{1}$ and (44). Note that equation (44) admits a solution $\alpha$ for $Q=\sqrt{r}$ iff $|f(\sqrt{r})| \leq s_{2 k}$. In the case $|f(\sqrt{r})|>2 s_{2 k}$ it follows immediately that $\mathcal{M}_{\gamma}^{-1}\left\{\left(r_{1}-r_{2}, 0\right)\right\}=\left\{\left(0,0, r_{1}, 0,0, r_{2}\right)\right\}$. If $|f(\sqrt{r})|=2 s_{2 k}$, then an analysis of the graph of $f$ near $(\sqrt{r}, f(\sqrt{r}))$ leads to the claimed result.

(ii) As $\lim _{q \backslash 0}|f(q)|=\infty$ it follows that there exists $-r_{1}<l_{\gamma, r}<r_{1}$ such that the interval $\left[l_{\gamma, r}, r_{1}\right]$ is a connected component of $(f \circ Q)^{-1}\left(\left[-2 s_{2 k}, 2 s_{2 k}\right]\right)$. It follows that for any $l_{\gamma, r} \leq L_{1} \leq r_{1}$ there exists a unique angle $0 \leq \alpha_{L_{1}} \leq \pi$ such that

$$
f\left(Q\left(L_{1}\right)\right)=-2 s_{2 k} \cos \alpha_{L_{1}} .
$$

The connected component of $\mathcal{M}_{\gamma}^{-1}\left\{\left(r_{1}-r_{2}, 0\right)\right\}$ containing $\left(0,0, r_{1}, 0,0, r_{2}\right)$ is then as claimed. Again by a straightforward computation $\left(0,0, r_{1}, 0,0, r_{2}\right)$ is a hyperbolic fixed point of $X_{\gamma}$. One can then argue as in the proof of Proposition 4.3 (ii) to show the remaining claims.

Proof of Theorem 4.1. Theorem 4.1 follows from Propositions 4.24 .4$.

It remains to study the critical points of $\mathcal{M}_{\gamma}$ with $\operatorname{rank}\left(d \mathcal{M}_{\gamma}\right)=1$, i.e. the points of $\left(\mathbb{S}_{r_{1}}^{2} \times \mathbb{S}_{r_{2}}^{2}\right) \backslash\left\{ \pm\left(0,0, r_{1}, 0,0, \pm r_{2}\right)\right\}$ where the vector fields $Y$ and $X_{\gamma}$ are collinear. In view of the formulas 377 for $Y$ and $X_{\gamma}$, points $\left(M_{i}, J_{i}, L_{i}\right) \in S_{r_{i}}^{2}, i=1$, 2, of this type have the property that the determinant of any $2 \times 2$-submatrix of the $2 \times 4$-matrix formed by $Y$ and $X_{\gamma}$ vanishes. This leads to the following system of equations:

$$
\begin{aligned}
& M_{1} J_{2}+M_{2} J_{1}=0, \\
& J_{1}^{2} L_{2}+L_{1} J_{2}^{2}-J_{1} J_{2}\left(d_{1, \gamma} L_{1}+d_{2, \gamma} L_{2}\right)=0, \\
& M_{1}^{2} L_{2}+L_{1} M_{2}^{2}+M_{1} M_{2}\left(d_{1, \gamma} L_{1}+d_{2, \gamma} L_{2}\right)=0, \\
& M_{1} J_{1} L_{2}-L_{1} M_{2} J_{2}+J_{1} M_{2}\left(d_{1, \gamma} L_{1}+d_{2, \gamma} L_{2}\right)=0 .
\end{aligned}
$$


Theorem 4.5. Assume that $1 \leq k<N / 4,0<r \leq 1$, and $\gamma \in \mathbb{R}$. If a point $\left(M_{i}, J_{i}, L_{i}\right)_{1 \leq i \leq 2} \in \mathbb{S}_{r_{1}}^{2} \times \mathbb{S}_{r_{2}}^{2} \backslash\left\{ \pm\left(0,0, r_{1}, 0,0, \pm r_{2}\right)\right\}$ is a critical point of $\mathcal{M}_{\gamma}$ with $\operatorname{rank}\left(d \mathcal{M}_{\gamma}\right)=1$ then $\left(M_{2}, L_{2}\right)=\lambda\left(M_{1},-J_{1}\right)$ for some $\lambda \in \mathbb{R}$, and

$\left(r_{1}^{2}-L_{1}^{2}\right)^{2} L_{2}^{2}+\left(r_{2}^{2}-L_{2}^{2}\right)^{2} L_{1}^{2}+2\left(r_{1}^{2}-L_{1}^{2}\right)\left(r_{2}^{2}-L_{2}^{2}\right)\left(2 L_{1} L_{2}-\left(d_{1, \gamma} L_{1}+d_{2, \gamma} L_{2}\right)^{2}\right)=0$.

Given any point $\left(M_{1}, J_{1}, L_{1}\right) \in \mathbb{S}_{r_{1}}^{2} \backslash\left\{ \pm\left(0,0, r_{1}\right)\right\}$ there exist at most eight points $\left(M_{2}, J_{2}, L_{2}\right) \in \mathbb{S}_{r_{2}}^{2} \backslash\left\{ \pm\left(0,0, r_{2}\right)\right\}$ such that $\left(M_{i}, J_{i}, L_{i}\right)_{1 \leq i \leq 2}$ is a critical point of $\mathcal{M}_{\gamma}$ with $\operatorname{rank}\left(d \mathcal{M}_{\gamma}\right)=1$.

Proof. First assume that $L_{1} \in\left\{ \pm r_{1}\right\}$. Then $J_{1}=M_{1}=0$. Hence (51) is automatically satisfied and equations (52) and (53) read $J_{2}=0$ and $M_{2}=0$, respectively. As a consequence, $\left(M_{1}, J_{1}, L_{1}\right)=\left(0,0, \pm r_{1}\right)$ and $\left(M_{2}, J_{2}, L_{2}\right)=\left(0,0, \pm r_{2}\right)$. In view of Theorem 4.1 we may thus assume that $\left|L_{1}\right|<r_{1}$. Then $\left(M_{1}, J_{1}\right) \neq(0,0)$. Hence the first equation (51) says that there exists $\lambda \in \mathbb{R}$ such that

$$
\left(M_{2}, J_{2}\right)=\lambda\left(M_{1},-J_{1}\right) .
$$

The conditions $\left(M_{i}, J_{i}, L_{i}\right) \in \mathbb{S}_{r_{i}}^{2}, i=1,2$, then imply that $\lambda$ satisfies

$$
\lambda^{2}=\frac{r_{2}^{2}-L_{2}^{2}}{r_{1}^{2}-L_{1}^{2}}
$$

Substituting (55) into 52)-54) one sees, again using $\left(M_{1}, J_{1}\right) \neq(0,0)$, that $52-54$ ) is equivalent to

$$
L_{2}+\lambda^{2} L_{1}+\lambda\left(d_{1, \gamma} L_{1}+d_{2, \gamma} L_{2}\right)=0
$$

or, taking squares, $\left(L_{2}+\lambda^{2} L_{1}\right)^{2}-\lambda^{2}\left(d_{1, \gamma} L_{1}+d_{2, \gamma} L_{2}\right)^{2}=0$. By 56, , the latter equation reads

$\left(r_{1}^{2}-L_{1}^{2}\right)^{2} L_{2}^{2}+\left(r_{2}^{2}-L_{2}^{2}\right)^{2} L_{1}^{2}+2\left(r_{1}^{2}-L_{1}^{2}\right)\left(r_{2}^{2}-L_{2}^{2}\right)\left(2 L_{1} L_{2}-\left(d_{1, \gamma} L_{1}+d_{2, \gamma} L_{2}\right)^{2}\right)=0$,

or, after dividing by $r_{1}^{2} r_{2}^{4}$ and using the bifurcation parameter $r$ and the normalized variables $l_{i}:=L_{i} / r_{i} \in(0,1)(i=1,2)$,

$r^{2}\left(1-l_{1}^{2}\right)^{2} l_{2}^{2}+\left(1-l_{2}^{2}\right)^{2} l_{1}^{2}+2 r\left(1-l_{1}^{2}\right)\left(1-l_{2}^{2}\right)\left(2 l_{1} l_{2}-\left(\sqrt{r} d_{1, \gamma} l_{1}+\frac{1}{\sqrt{r}} d_{2, \gamma} l_{2}\right)^{2}\right)=0$.

Note that for given $r$ and $0<l_{1}<1$, the left hand side of (58) is a polynomial in $l_{2}$ of degree four, $\left(l_{1}^{2}+d_{2, \gamma}^{2}\left(1-l_{1}^{2}\right)\right) l_{2}^{4}+O\left(l_{2}^{3}\right)$.

Summarizing, we have shown that for any given point $\left(M_{1}, J_{1}, L_{1}\right) \in \mathbb{S}_{r_{1}}^{2} \backslash$ $\left\{\left(0,0, \pm r_{1}\right)\right\}$, there exist at most eight points $\left(M_{2}, J_{2}, L_{2}\right) \in \mathbb{S}_{r_{2}}^{2} \backslash\left\{\left(0,0, \pm r_{2}\right)\right\}$ such that $Y$ and $X_{\gamma}$ are collinear. Indeed, for any $\left(M_{1}, J_{1}, L_{1}\right) \in \mathbb{S}_{r_{1}}^{2} \backslash\left\{\left(0,0, \pm r_{1}\right)\right\}$ a solution $\left(M_{2}, J_{2}, L_{2}\right) \in \mathbb{S}_{r_{2}}^{2} \backslash\left\{\left(0,0, \pm r_{2}\right)\right\}$ of $\left.51-\sqrt{54}\right)$ is given by $\left(M_{2}, J_{2}\right)=\lambda\left(M_{1}, J_{1}\right)$ and $L_{2}=r_{2} l_{2}$ with $\lambda$ and $l_{2}$ satisfying (56), respectively (58). As a consequence, the set of solutions of 51)-54 is an algebraic subset of $\mathbb{S}_{r_{1}}^{2} \times \mathbb{S}_{r_{2}}^{2}$ of dimension at most two. 


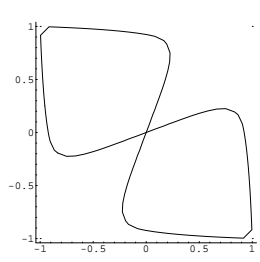

(a) $k=1$

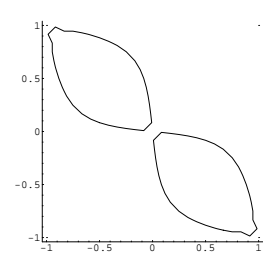

(b) $k=3$

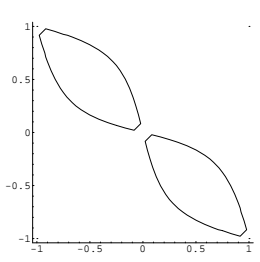

(c) $k=5$

Fig. 2. Sets of solutions $\left(l_{1}, l_{2}\right)$ of 58 for $N=24, r=1, \gamma=-0.5$.

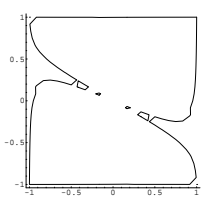

(a) $k=1$

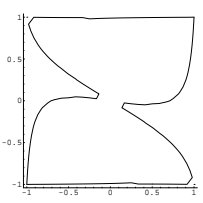

(b) $k=3$

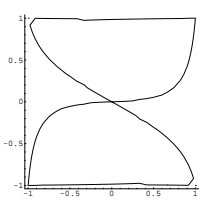

(c) $k=5$

Fig. 3. Sets of solutions $\left(l_{1}, l_{2}\right)$ of 58 for $N=24, r=0.3, \gamma=-2.5$.

In order to analyze the critical points of $\mathcal{M}_{\gamma}$ with $\operatorname{rank}\left(d \mathcal{M}_{\gamma}\right)=1$, we perform another symplectic reduction. First we pass to the orbit space of the flow of $Y$ on $\mathbb{S}_{r_{1}}^{2} \times \mathbb{S}_{r_{2}}^{2}$ and then to the level sets of $G$.

In view of 39 , the $Y$-flow is an $\mathbb{S}^{1}$-action. Note that besides $L_{1}$ and $L_{2}$, the quantities $\sigma$ and $\tau$ are invariant under this $\mathbb{S}^{1}$-action, where

$$
\sigma:=M_{1} M_{2}-J_{1} J_{2}, \quad \tau:=M_{1} J_{2}+M_{2} J_{1}
$$

They are related by the identity

$$
\sigma^{2}+\tau^{2}=\prod_{i=1}^{2}\left(r_{i}^{2}-L_{i}^{2}\right)
$$

Define

$$
\mathcal{F}^{(3)}: \dot{\mathbb{S}}_{r_{1}}^{2} \times \dot{\mathbb{S}}_{r_{2}}^{2} \rightarrow \mathbb{R}^{4}, \quad\left(M_{1}, J_{1}, L_{1}, M_{2}, J_{2}, L_{2}\right) \mapsto\left(L_{1}, L_{2}, \sigma, \tau\right),
$$

where $\dot{\mathbb{S}}_{r_{i}}^{2}:=\mathbb{S}_{r_{i}}^{2} \backslash\left\{\left(0,0, \pm r_{i}\right)\right\}$. Let $\mathcal{O}_{r}$ denote the image of $\mathcal{F}^{(3)}$. For any element $\left(L_{1}, L_{2}, s, t\right) \in \mathcal{O}_{r}$ we have

$$
s^{2}+t^{2}=\prod_{i=1}^{2}\left(r_{i}^{2}-L_{i}^{2}\right) \quad \text { and } \quad\left|L_{i}\right| \leq r_{i}(i=1,2) .
$$


The fibers of $\mathcal{F}^{(3)}$ are the orbits of the $Y$-action on $\dot{\mathbb{S}}_{r_{1}}^{2} \times \dot{\mathbb{S}}_{r_{2}}^{2}$, i.e. $\mathcal{O}_{r}$ coincides with the orbit space of the $Y$-action on $\dot{\mathbb{S}}_{r_{1}}^{2} \times \dot{\mathbb{S}}_{r_{2}}^{2}$. As a consequence, any function on $\dot{\mathbb{S}}_{r_{1}}^{2} \times \dot{\mathbb{S}}_{r_{2}}^{2}$ which Poisson commutes with $G$ factors through $\mathcal{O}_{r}$.

In particular, $K_{\gamma}$ and $G$ factor through $\mathcal{O}_{r}$. In fact, $K_{\gamma}$ and $G$, when expressed in the variables $L_{1}, L_{2}, \sigma, \tau$, are polynomials, given by

$$
K_{\gamma}=\sum_{i=1}^{2} \frac{1}{2} d_{i, \gamma}\left(r_{i}^{2}-L_{i}^{2}\right)+\sigma, \quad G=L_{1}-L_{2} .
$$

By reducing the system $\left(G, K_{\gamma}\right)$ by the $Y$-action one obtains a family of integrable systems with one degree of freedom parametrized by the value $c$ of $G$. Denote by $X_{\gamma, c}$ the Hamiltonian vector field induced by $K_{\gamma}$. The fixed points of $X_{\gamma, c}$ can then be characterized in terms of the bifurcation parameters $\gamma, r$, and $k$.

Note that by (51), the rank-1-points of the reduced moment map $\mathcal{M}_{\gamma}$ satisfy $\tau=0$, and by $55-56, \sigma^{2}=\left(r_{2}^{2}-L_{2}^{2}\right)\left(r_{1}^{2}-L_{1}^{2}\right)$. Hence the image of the set of the rank-1points under $\mathcal{F}^{(3)}$ is an algebraic subset of $\mathcal{O}_{r}$ of dimension at most one-see 58 .

By $59,, \sigma$ and $\tau$ are located on a circle of radius $\sqrt{\left(r_{1}^{2}-L_{1}^{2}\right)\left(r_{2}^{2}-L_{2}^{2}\right)}$,

$$
(\sigma, \tau)=\sqrt{\left(r_{1}^{2}-L_{1}^{2}\right)\left(r_{2}^{2}-L_{2}^{2}\right)}(\cos \phi, \sin \phi),
$$

where $\phi \in \mathbb{R} / 2 \pi \mathbb{Z}$. The phase spaces, reduced by the $Y$-action, are now obtained by taking subsets of $\mathcal{O}_{r}$ corresponding to level sets of $G$, i.e. by replacing $L_{2}$ by $L_{1}-c$, where $c$ is the value of $G$. The restriction $K_{\gamma, c}$ of $K_{\gamma}$ to the reduced phase space corresponding to the value $c$ of $G$ is then given by

$$
\begin{aligned}
K_{\gamma, c}\left(L_{1}, \phi\right)= & \frac{1}{2}\left(d_{1, \gamma}\left(r_{1}^{2}-L_{1}^{2}\right)+d_{2, \gamma}\left(r_{2}^{2}-\left(L_{1}-c\right)^{2}\right)\right) \\
& +\sqrt{\left(r_{1}^{2}-L_{1}^{2}\right)\left(r_{2}^{2}-\left(L_{1}-c\right)^{2}\right)} \cos \phi
\end{aligned}
$$

with $L_{1} \in\left(-r_{1}, r_{1}\right) \cap\left(c-r_{2}, c+r_{2}\right)$ and $\phi \in \mathbb{R} / 2 \pi \mathbb{Z}$.

The reduced Hamiltonian vector field induced by $K_{\gamma, c}$ is given by

$$
X_{\gamma, c}\left(L_{1}, \phi\right)=\frac{d}{d t}\left(\begin{array}{c}
L_{1} \\
\phi
\end{array}\right)=\left\{L_{1}, \phi\right\}\left(\begin{array}{c}
\partial K_{\gamma, c} / \partial \phi \\
-\partial K_{\gamma, c} / \partial L_{1}
\end{array}\right)
$$

Note that

$$
\begin{aligned}
\left\{L_{1}, \phi\right\} & =\left\{L_{1}, \arctan \frac{\tau}{\sigma}\right\}=\frac{1}{1+(\tau / \sigma)^{2}}\left\{L_{1}, \frac{\tau}{\sigma}\right\} \\
& =\frac{1}{1+(\tau / \sigma)^{2}} \cdot \frac{\sigma\left\{L_{1}, \tau\right\}-\tau\left\{L_{1}, \sigma\right\}}{\sigma^{2}}=\frac{1}{1+(\tau / \sigma)^{2}} \cdot \frac{\sigma^{2}+\tau^{2}}{\sigma^{2}}=1,
\end{aligned}
$$


since $\left\{L_{1}, \tau\right\}=\sigma$ and $\left\{L_{1}, \sigma\right\}=-\tau$. Furthermore, with $L_{2}=L_{1}-c$,

$$
\begin{aligned}
& \frac{\partial K_{\gamma, c}}{\partial \phi}=-\sqrt{\left(r_{1}^{2}-L_{1}^{2}\right)\left(r_{2}^{2}-L_{2}^{2}\right)} \sin \phi, \\
& \frac{\partial K_{\gamma, c}}{\partial L_{1}}=-\left(d_{1, \gamma} L_{1}+d_{2, \gamma} L_{2}\right)-\frac{L_{1}\left(r_{2}^{2}-L_{2}^{2}\right)+L_{2}\left(r_{1}^{2}-L_{1}^{2}\right)}{\sqrt{\left(r_{1}^{2}-L_{1}^{2}\right)\left(r_{2}^{2}-L_{2}^{2}\right)}} \cos \phi .
\end{aligned}
$$

Hence (63) reads

$$
X_{\gamma, c}\left(L_{1}, \phi\right)=\left(\begin{array}{c}
-\sqrt{\left(r_{1}^{2}-L_{1}^{2}\right)\left(r_{2}^{2}-L_{2}^{2}\right)} \sin \phi \\
\left(d_{1, \gamma} L_{1}+d_{2, \gamma} L_{2}\right)+\frac{L_{1}\left(r_{2}^{2}-L_{2}^{2}\right)+L_{2}\left(r_{1}^{2}-L_{1}^{2}\right)}{\sqrt{\left(r_{1}^{2}-L_{1}^{2}\right)\left(r_{2}^{2}-L_{2}^{2}\right)}} \cos \phi
\end{array}\right),
$$

where we treat $L_{2}=L_{1}-c$ as a dependent variable.

By (64), the fixed points of the vector field $X_{\gamma, c}$ with $\left|L_{1}\right|<r_{1}$ are given by $\left(L_{1}, \phi\right)$ satisfying

$$
\phi \in \pi \mathbb{Z}
$$

and

$$
\left(d_{1, \gamma} L_{1}+d_{2, \gamma} L_{2}\right)+\frac{L_{1}\left(r_{2}^{2}-L_{2}^{2}\right)+L_{2}\left(r_{1}^{2}-L_{1}^{2}\right)}{\sqrt{\left(r_{1}^{2}-L_{1}^{2}\right)\left(r_{2}^{2}-L_{2}^{2}\right)}} \cos \phi=0 .
$$

Note that $(65)$ and the square of $(66)$ are equivalent to the system of equations 51$]-58$ derived above.

In order to determine the type of the fixed points $\left(L_{1}, \phi\right)$, i.e. points satisfying 65)(66) for a given value $c$ of $G$, one computes the Jacobian of $X_{\gamma, c}, H \equiv H_{\gamma, c}\left(L_{1}, \phi\right)=$ $\left(\begin{array}{ll}h_{11} & h_{12} \\ h_{21} & h_{22}\end{array}\right)$ at these points. Note that at such points $h_{11}=0$ and $h_{22}=0$ and thus $\operatorname{det}(H)=$ $-h_{12} h_{21}$. Hence such a fixed point is an elliptic or hyperbolic fixed point of $X_{\gamma, c}$ if $h_{12} h_{21}$ is positive, respectively negative. We omit a more detailed analysis of these points.

\section{Chains with Dirichlet boundary conditions}

In this section we consider a FPU chain with $N^{\prime}\left(N^{\prime} \geq 3\right.$, not necessarily even) moving particles and fixed endpoints, i.e. with boundary conditions [18].

It has been observed that such a chain can be treated as an invariant subsystem of a periodic lattice with $N=2 N^{\prime}+2$ particles—see [13]: Let $T^{*} \mathbb{R}^{N}$ be endowed with the canonical symplectic structure and consider the linear map $S: T^{*} \mathbb{R}^{N} \rightarrow T^{*} \mathbb{R}^{N}$ sending $\left(q_{i}\right)_{1 \leq i \leq N},\left(p_{i}\right)_{1 \leq i \leq N}$ to

$$
-\left(q_{N-1}, \ldots, q_{1}, q_{N}\right),-\left(p_{N-1}, \ldots, p_{1}, p_{N}\right) .
$$

Then $S$ is a canonical linear involution satisfying $H_{V} \circ S=H_{V}$. Denote by Fix $(S)$ the fixed point set of $S$. Then $\operatorname{Fix}(S)$ is the subset of all elements $(q, p)$ in $T^{*} \mathbb{R}^{N}$ satisfying

$$
\left(q_{n}, p_{n}\right)=-\left(q_{N-n}, p_{N-n}\right) \quad \forall 1 \leq n \leq N-1 \quad \text { and } \quad q_{N}=p_{N}=0 .
$$


In particular, on $\operatorname{Fix}(S), q_{N}=q_{N^{\prime}+1}=0$ and $p_{N}=p_{N^{\prime}+1}=0$. Note that on $\operatorname{Fix}(S)$, both the center of mass coordinate $Q=N^{-1} \sum_{i=1}^{N} q_{i}$ and its momentum $P=N^{-1} \sum_{i=1}^{N} p_{i}$ are identically 0 . Hence $\operatorname{Fix}(S) \subseteq \mathcal{M}$, where

$$
\mathcal{M}:=\left\{(q, p) \in T^{*} \mathbb{R}^{N} \mid Q=0, P=0\right\} .
$$

We endow $\mathcal{M}$ with the symplectic structure induced from $T^{*} \mathbb{R}^{N}$.

The phase space of an FPU chain with $N^{\prime}$ moving particles satisfying Dirichlet boundary conditions is $T^{*} \mathbb{R}^{N^{\prime}}$, endowed with the canonical symplectic structure $\sum_{i=1}^{N^{\prime}} d q_{i} \wedge$ $d p_{i}$. It can be embedded into $\mathcal{M}$ by the map $\Theta: T^{*} \mathbb{R}^{N^{\prime}} \rightarrow \mathcal{M}$ defined by

$$
\left(q_{i}, p_{i}\right)_{1 \leq i \leq N^{\prime}} \mapsto \frac{1}{\sqrt{2}}\left(\left(q_{i}, p_{i}\right)_{1 \leq i \leq N^{\prime}},(0,0),-\left(q_{N^{\prime}-i}, p_{N^{\prime}-i}\right)_{0 \leq i \leq N^{\prime}-1},(0,0)\right) .
$$

Note that $\Theta\left(T^{*} \mathbb{R}^{N^{\prime}}\right)=\operatorname{Fix}(S)$, i.e. $\Theta$ is a parametrization of $\operatorname{Fix}(S)$ and the pullback of the canonical symplectic form on $\mathcal{M}$ by $\Theta$ is $\sum_{i=1}^{N^{\prime}} d q_{i} \wedge d p_{i}$, which means that $\Theta$ is canonical. It then follows that $\operatorname{Fix}(S)$ is a symplectic submanifold of $\mathcal{N}$.

We now express the equations defining $\operatorname{Fix}(S)$ locally near 0 as a subset of $\mathcal{M}$ in terms of the canonical coordinates $\left(x_{k}, y_{k}\right)_{1 \leq k \leq N-1}$ provided by Theorem 1.1 or even more conveniently, in terms of the associated complex coordinates $\left(\zeta_{k}\right)_{1 \leq|k| \leq N-1}$, defined for $1 \leq k \leq N-1$ by

$$
\zeta_{k}=\frac{1}{\sqrt{2}}\left(x_{k}-i y_{k}\right), \quad \zeta_{-k}=\overline{\zeta_{k}}=\frac{1}{\sqrt{2}}\left(x_{k}+i y_{k}\right)
$$

Denote by $Z$ the linear subspace of $\mathbb{C}^{2 N-2}$ consisting of such vectors $\left(\zeta_{k}\right)_{1 \leq|k| \leq N-1}$. Below we also write $\left(\zeta_{k}\right)_{1 \leq k \leq N-1}$ for the element $\left(\zeta_{k}\right)_{1 \leq|k| \leq N-1} \in Z$ and use the notations $(n \in \mathbb{Z})$

Define the map $S_{z}: Z \rightarrow Z$ by

$$
c_{n}:=\cos \frac{n \pi}{N}, \quad s_{n}:=\sin \frac{n \pi}{N} .
$$

$$
\left(\zeta_{k}\right)_{1 \leq k \leq N-1} \mapsto\left(-e^{4 \pi i k / N} \zeta_{N-k}\right)_{1 \leq k \leq N-1} .
$$

Like the map $S: \mathcal{M} \rightarrow \mathcal{M}, S_{\mathcal{Z}}$ is a canonical linear involution. In fact, the maps $S$ and $S_{Z}$ are conjugate to each other under the coordinate change of Theorem 1.1. Before making this statement more precise, let us introduce a parametrization of the fixed point set Fix $\left(S_{z}\right)$. Introduce

$$
Z_{\text {Dir }}:=\left\{\left(\zeta_{k}\right)_{1 \leq|k| \leq N^{\prime}} \in \mathbb{C}^{2 N^{\prime}} \mid \overline{\zeta_{k}}=\zeta_{-k} \forall 1 \leq k \leq N^{\prime}\right\},
$$

endowed with the canonical symplectic structure induced from $\mathbb{C}^{2 N^{\prime}}$, and the embedding $\Theta_{Z}: Z_{\text {Dir }} \rightarrow Z$ mapping $\left(\zeta_{k}\right)_{1 \leq|k| \leq N^{\prime}}$ to the element $\left(\tilde{\zeta}_{k}\right)_{1 \leq k \leq N-1} \in \mathcal{Z}$ given by

$$
\frac{1}{\sqrt{2}}\left(\left(\zeta_{k}\right)_{1 \leq k \leq N^{\prime}}, 0,\left(-e^{4 \pi i k / N} \zeta_{N^{\prime}+1-k}\right)_{1 \leq k \leq N^{\prime}}\right)
$$

Note that $\Theta_{z}\left(Z_{\text {Dir }}\right)=\operatorname{Fix}\left(S_{z}\right)$, i.e. $\Theta_{z}$ is a parametrization of $\operatorname{Fix}\left(S_{Z}\right)$.

Using the explicit construction in [7] of the canonical transformation $\Psi$ of Theorem 1.1 one verifies the following lemma. 
Lemma 5.1. In terms of the complex variables $\left(\zeta_{k}\right)_{1 \leq|k| \leq N-1}$ defined by Theorem 1.1 . near 0 , the map $S$ is given by $S_{Z}$. More precisely, if $\Psi$, defined near $0 \in Z$, is the coordinate transformation given by Theorem 1.1. then $S \circ \Psi=\Psi \circ S_{z}$. In particular, locally near 0 , the set $\operatorname{Fix}\left(S_{Z}\right) \subseteq Z$, described by the equations

$$
e^{-2 \pi i k / N} \zeta_{k}+e^{2 \pi i k / N} \zeta_{N-k}=0 \quad(1 \leq k \leq N-1),
$$

is the image of $\operatorname{Fix}(S)$ under $\Psi^{-1}$. Expressed in terms of the real variables $\left(x_{k}, y_{k}\right)_{1 \leq k \leq N-1}$, the conditions 69 are given by

$$
\left(\begin{array}{cc}
c_{2 k} & -s_{2 k} \\
s_{2 k} & c_{2 k}
\end{array}\right)\left(\begin{array}{l}
x_{k} \\
y_{k}
\end{array}\right)+\left(\begin{array}{cc}
c_{2 k} & s_{2 k} \\
-s_{2 k} & c_{2 k}
\end{array}\right)\left(\begin{array}{l}
x_{N-k} \\
y_{N-k}
\end{array}\right)=\left(\begin{array}{l}
0 \\
0
\end{array}\right) .
$$

In particular, for $k=N^{\prime}+1(=N / 2)$ we get $\zeta_{N^{\prime}+1}=0$ and therefore

$$
\left(x_{N^{\prime}+1}, y_{N^{\prime}+1}\right)=(0,0) \text {. }
$$

Corollary 5.2. On $\operatorname{Fix}\left(S_{z}\right)$, for any $1 \leq k \leq N / 2$,

$$
I_{k}=I_{N-k}
$$

and

$$
J_{k} J_{N / 2-k}-M_{k} M_{N / 2-k}=I_{k} I_{N / 2-k}
$$

Moreover,

$$
I_{N / 2}=0
$$

Proof. In terms of the complex variables $\left(\zeta_{k}\right)_{1 \leq|k| \leq N-1}, I_{k}=\zeta_{k} \zeta_{-k}$ for any $1 \leq k \leq$ $N-1$. Hence on Fix $\left(S_{z}\right)$,

$$
I_{k}=\zeta_{k} \zeta_{-k}=\left(-e^{4 \pi i k / N} \zeta_{N-k}\right)\left(-e^{-4 \pi i k / N} \zeta_{-(N-k)}\right)=\zeta_{N-k} \zeta_{-(N-k)}=I_{N-k},
$$

showing (71). The identity (73) follows from $\left.\zeta_{N / 2}\right|_{\text {Fix }\left(S_{z}\right)}=0$. To prove (72), we first conclude from 69 that on $\operatorname{Fix}\left(S_{Z}\right)$, for any $1 \leq k \leq N-1$,

$$
J_{k}=-c_{4 k} I_{k}, \quad M_{k}=-s_{4 k} I_{k} .
$$

Hence on $\operatorname{Fix}\left(S_{Z}\right)$,

$$
\begin{aligned}
J_{k} J_{N / 2-k}-M_{k} M_{N / 2-k} & =I_{k} I_{N / 2-k}\left(c_{4 k} c_{4(N / 2-k)}-s_{4 k} s_{4(N / 2-k)}\right) \\
& =I_{k} I_{N / 2-k}\left(c_{4 k}^{2}+s_{4 k}^{2}\right)=I_{k} I_{N / 2-k} .
\end{aligned}
$$

From the definitions (6), (11), and (12) of the variables $I_{k}, J_{k}, M_{k}$, and of the expressions $R$ and $R_{N / 4}$ one then obtains the following

Corollary 5.3. On $\operatorname{Fix}\left(S_{z}\right)$,

$$
R=4 \sum_{1 \leq k<N / 4} s_{2 k} I_{k} I_{N / 2-k} \text { and } \quad R_{N / 4}= \begin{cases}I_{N / 4}^{2} & \text { if } N / 4 \in \mathbb{N} \\ 0 & \text { otherwise }\end{cases}
$$


It follows from Corollary 5.3 that on $\operatorname{Fix}\left(S_{\mathcal{Z}}\right)$, the expression 13$]$ is in Birkhoff normal form up to order 4 . This allows us to prove Theorem 1.3 .

Proof of Theorem 1.3. We start with the resonant normal form (13) for even chains, $N P^{2} / 2+H_{\alpha, \beta}(I)-R_{\alpha, \beta}(J, M)+O\left(|(x, y)|^{5}\right)$, where $H_{\alpha, \beta}(I)$ and $R_{\alpha, \beta}(J, M)$ are given by $(8)$ and $(10)$, respectively. In view of the identity $I_{k}=I_{N-k}$, the terms in the decomposition 14 of $H_{\alpha, \beta}$, when restricted to $\operatorname{Fix}\left(S_{z}\right)$, are given by

$$
\begin{gathered}
H^{(2)}(I)=4 \sum_{k=1}^{N^{\prime}} s_{k} I_{k}, \\
H_{\alpha, \beta}^{(4)}(I)=\frac{1}{N} \sum_{k=1}^{N^{\prime}} d_{k}^{+} I_{k}^{2}+\frac{4\left(\beta-\alpha^{2}\right)}{2 N} \sum_{\substack{1 \leq k, l \leq N^{\prime} \\
k \neq l}} s_{k} s_{l} I_{k} I_{l},
\end{gathered}
$$

and

$$
\frac{1}{2 N} \sum_{k=1}^{N / 2-1} d_{k}^{-} I_{k} I_{N-k}=\frac{1}{2 N} \sum_{k=1}^{N^{\prime}} d_{k}^{-} I_{k}^{2} .
$$

From Corollary 5.3 , we conclude that on $\operatorname{Fix}\left(S_{z}\right)$,

$$
\begin{aligned}
-R_{\alpha, \beta}(J, M) & =-\frac{\beta-\alpha^{2}}{4 N}\left(R(J, M)+R_{N / 4}(J, M)\right) \\
& =-\frac{\beta-\alpha^{2}}{4 N}(4 \sum_{1 \leq k<N / 4} s_{2 k} I_{k} I_{N / 2-k} \underbrace{+I_{N / 4}^{2}}_{\text {only if } N / 4 \in \mathbb{N}}) .
\end{aligned}
$$

Formula $\sqrt{19}$ is then obtained by adding up $74-\sqrt{77})$, noting that $d_{k}^{+}+d_{k}^{-} / 2=\frac{1}{2}\left(\alpha^{2}+\right.$ $3\left(\beta-\alpha^{2}\right) s_{k}^{2}$, and replacing $I_{k}$ by its pullback $\frac{1}{2} I_{k}$ with respect to the parametrization $\Theta_{z}$ of Fix $\left(S_{Z}\right)$ introduced above.

It remains to prove Theorem 1.5 . We first consider the case $\alpha=0$.

Proposition 5.4. Assume that $\alpha=0$ in (3). Then the following holds:

(i) The Birkhoff normal form of $H_{V}$ with Dirichlet boundary conditions up to order 4 is given by $\left(N^{\prime}+1\right) P^{2} / 2+H_{0, \beta}^{D}(I)$ where

$$
\begin{gathered}
H_{0, \beta}^{D}(I)=2 \sum_{k=1}^{N^{\prime}} s_{k} I_{k}+\frac{\beta}{16\left(N^{\prime}+1\right)}(\sum_{k=1}^{N^{\prime}} 3 s_{k}^{2} I_{k}^{2} \underbrace{+\frac{1}{2} I_{\left(N^{\prime}+1\right) / 2}^{2}}_{\text {only if } \frac{N^{\prime}+1}{2} \in \mathbb{N}} \\
\left.+4 \sum_{\substack{l \neq m \\
1 \leq l, m \leq N^{\prime}}} s_{l} s_{m} I_{l} I_{m}-\sum_{k=1}^{N^{\prime}} s_{2 k} I_{k} I_{N^{\prime}+1-k}\right) .
\end{gathered}
$$

(ii) For any $\beta \neq 0, H_{0, \beta}^{D}(I)$ is nondegenerate at $I=0$. 
Proof. The Birkhoff normal form (78) of $H_{V}$ with Dirichlet boundary conditions is given by the formula $\left[19\right.$ evaluated at $\alpha=0$. To investigate the Hessian of $Q_{0, \beta}^{D}$ of $H_{0, \beta}^{D}(I)$ at $I=0$, we write

$$
Q_{0, \beta}^{D}=\frac{2 \beta}{16\left(N^{\prime}+1\right)} \Delta^{N^{\prime}} P^{D} \Delta^{N^{\prime}}
$$

where $\Delta^{N^{\prime}}=\operatorname{diag}\left(\sin \frac{k \pi}{2 N^{\prime}+2}\right)_{1 \leq k \leq N^{\prime}}$ and $P^{D}$ is the $N^{\prime} \times N^{\prime}$-matrix which for $N^{\prime}$ even resp. odd is of the form

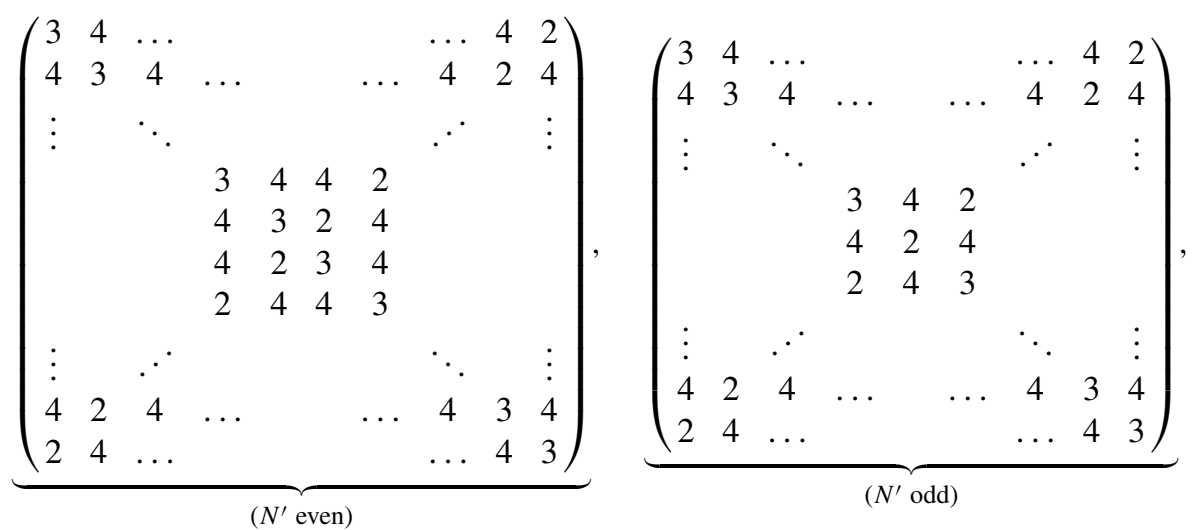

where we have used the fact that $s_{2 k}=2 s_{k} c_{k}=2 s_{k} s_{N^{\prime}+1-k}$ and, if $\left(N^{\prime}+1\right) / 2 \in \mathbb{N}$, $s_{\left(N^{\prime}+1\right) / 2}^{2}=1 / 2$. It follows that

$$
\operatorname{det}\left(Q_{0, \beta}^{D}\right)=\left(\frac{2 \beta}{16\left(N^{\prime}+1\right)}\right)^{N-1} \cdot \operatorname{det}\left(P^{D}\right) \cdot \prod_{k=1}^{N^{\prime}} \sin ^{2} \frac{k \pi}{2 N^{\prime}+2}
$$

In order to see that $P^{D}$ is nonsingular, observe that $\operatorname{det}\left(P^{D}\right) \in \mathbb{Z}$. For $N^{\prime}$ even we show that $\operatorname{det}\left(P^{D}\right) \equiv 1 \bmod 2$. Note that in this case the diagonal of $P^{D}$ consists of 3's only. Therefore $\operatorname{det}\left(P^{D}\right) \equiv 3^{N^{\prime}} \bmod 2 \equiv 1 \bmod 2$. If $N^{\prime}$ is odd, the same argument shows that $\operatorname{det}\left(P^{D}\right) \equiv 2 \bmod 4$. Hence, if $\beta \neq 0$, then $\operatorname{det}\left(Q_{0, \beta}^{D}\right) \neq 0$, and the nondegeneracy of the Hessian of $H_{0, \beta}^{D}(I)$ at $I=0$ follows.

Lemma 5.5. If $\beta<0$, then $Q_{0, \beta}^{D}$ has $\left\lceil\left(N^{\prime}+1\right) / 2\right\rceil$ negative eigenvalues, whereas if $\beta>0$, then $Q_{0, \beta}^{D}$ has $\left\lfloor\left(N^{\prime}-1\right) / 2\right\rfloor$ negative eigenvalues. In particular, for any $\beta \neq 0$, $Q_{0, \beta}^{D}$ is indefinite (and $H_{0, \beta}^{D}$ is therefore not convex).

Proof. We want to use the decomposition 79 of $Q_{0, \beta}^{D}$ to show that $Q_{0, \beta}^{D}$ can be deformed continuously to $\frac{2 \beta}{16\left(N^{\prime}+1\right)} P^{D}$ : Consider, for $0 \leq t \leq 1$,

$$
Q_{0, \beta}^{D}(t):=\frac{2 \beta}{16\left(N^{\prime}+1\right)}\left(t \Delta^{N^{\prime}}+(1-t) \operatorname{Id}\right) P^{D}\left(t \Delta^{N^{\prime}}+(1-t) \operatorname{Id}\right) .
$$


As $t \Delta^{N^{\prime}}+(1-t)$ Id is positive definite for any $0 \leq t \leq 1$ and $P^{D}$ is regular and symmetric, $Q_{0, \beta}^{D}(t)$ is a symmetric regular $N^{\prime} \times N^{\prime}$-matrix for any $0 \leq t \leq 1$. For $t=0$, $Q_{0, \beta}^{D}(0)=\frac{2 \beta}{16\left(N^{\prime}+1\right)} P^{D}$, whereas for $t=1, Q_{0, \beta}^{D}(1)=Q_{0, \beta}^{D}$. Therefore, index $\left(Q_{0, \beta}^{D}\right)$ (i.e. the number of negative eigenvalues of $\left.Q_{0, \beta}^{D}\right)$ coincides with index $\left(\frac{2 \beta}{16\left(N^{\prime}+1\right)} P^{D}\right)$. To describe the spectrum of $P^{D}$, we distinguish between $N^{\prime}$ even and odd.

If $N^{\prime}$ is even, the eigenvalues of $P^{D}$ are $4 N^{\prime}-3$ (with multiplicity one), 1 (with multiplicity $N^{\prime} / 2$ ), and -3 (with multiplicity $N^{\prime} / 2-1$ ), hence $P^{D}$ has $N^{\prime} / 2-1$ negative eigenvalues. If $N^{\prime}$ is odd, the eigenvalues of $P^{D}$ are 1 (with multiplicity $\left.\left(N^{\prime}-1\right) / 2\right),-3$ (with multiplicity $\left.\left(N^{\prime}-3\right) / 2\right)$, and $\frac{1}{2}\left(4 N^{\prime}-5\right)\left(1 \pm \sqrt{1+\frac{8\left(4 N^{\prime}-1\right)}{\left(4 N^{\prime}-5\right)^{2}}}\right)$ (each with multiplicity one), hence $P^{D}$ has $\left(N^{\prime}-1\right) / 2$ negative eigenvalues. These facts are verified in Appendix A. The claim of the lemma now follows.

We now turn to the case $\alpha \neq 0$.

Proposition 5.6. Assume that $\alpha \neq 0$ in 3. Then, for $\alpha$ fixed, $\operatorname{det}\left(Q_{\alpha, \beta}^{D}\right)$ is a polynomial in $\beta$ of degree $N^{\prime}$ and has $N^{\prime}$ real zeroes (counted with multiplicities). When denoted by $\beta_{k}=\beta_{k}(\alpha)\left(1 \leq k \leq N^{\prime}\right)$ and listed in increasing order, they satisfy

$$
\beta_{1} \leq \cdots \leq \beta_{\left\lceil\left(N^{\prime}+1\right) / 2\right\rceil}<\alpha^{2}<\beta_{\left\lceil\left(N^{\prime}+3\right) / 2\right\rceil} \leq \cdots \leq \beta_{N^{\prime}} .
$$

Moreover,

$$
\operatorname{index}\left(Q_{\alpha, \beta}^{D}\right)= \begin{cases}\left\lceil\left(N^{\prime}+1\right) / 2\right\rceil & \text { for } \beta<\beta_{1}, \\ 0 & \text { for } \beta_{\left\lceil\left(N^{\prime}+1\right) / 2\right\rceil}<\beta<\beta_{\left\lceil\left(N^{\prime}+3\right) / 2\right\rceil}, \\ \left\lfloor\left(N^{\prime}-1\right) / 2\right\rfloor & \text { for } \beta>\beta_{N^{\prime}},\end{cases}
$$

Proof. Fix $\alpha \in \mathbb{R} \backslash\{0\}$ and consider the map $\beta \mapsto \operatorname{det}\left(Q_{\alpha, \beta}^{D}\right)$. It follows from 19 that $\operatorname{det}\left(Q_{\alpha, \beta}^{D}\right)$ is a polynomial in $\beta$ of degree at most $N^{\prime}$,

$$
\operatorname{det}\left(Q_{\alpha, \beta}^{D}\right)=\sum_{j=0}^{N^{\prime}} r_{j} \beta^{j}
$$

where $r_{0}=\operatorname{det}\left(Q_{\alpha, 0}^{D}\right)$ and $r_{N^{\prime}}=\operatorname{det}\left(Q_{0,1}^{D}\right)$. By Proposition 5.4, $\operatorname{det}\left(Q_{0,1}^{D}\right) \neq 0$, hence the degree of the polynomial $\operatorname{det}\left(Q_{\alpha, \beta}^{D}\right)$ is $N^{\prime}$. We claim that $\operatorname{det}\left(Q_{\alpha, \beta}^{D}\right)$ has $N^{\prime}$ real zeroes (counted with multiplicities). For $|\beta|$ large enough, $\operatorname{index}\left(Q_{\alpha, \beta}^{D}\right)$ is equal to index $\left(Q_{0, \beta}^{D}\right)$. By Lemma 5.5 index $\left(Q_{0, \beta}^{D}\right)$ is $\left\lfloor\left(N^{\prime}-1\right) / 2\right\rfloor$ for $\beta>0$ and $\left\lceil\left(N^{\prime}+1\right) / 2\right\rceil$ for $\beta<0$. Hence there exists $R>0$ such that index $\left(Q_{\alpha, \beta}^{D}\right)=\left\lfloor\left(N^{\prime}-1\right) / 2\right\rfloor$ for any $\beta>R$ and index $\left(Q_{\alpha, \beta}^{D}\right)=\left\lceil\left(N^{\prime}+1\right) / 2\right\rceil$ for any $\beta<-R$. For $\beta=\alpha^{2}, Q_{\alpha, \alpha^{2}}^{D}$ is a positive multiple of the identity matrix, hence index $\left(Q_{\alpha, \alpha^{2}}^{D}\right)=0$. It then follows that, when counted with multiplicities, index $\left(Q_{\alpha, \beta}^{D}\right)$ must change at least $\left\lceil\left(N^{\prime}+1\right) / 2\right\rceil$ times in the open interval $\left(-\infty, \alpha^{2}\right)$ and at least $\left\lfloor\left(N^{\prime}-1\right) / 2\right\rfloor$ times in $\left(\alpha^{2}, \infty\right)$. Since a change of index $\left(Q_{\alpha, \beta}^{D}\right)$ induces a real zero of $\operatorname{det}\left(Q_{\alpha, \beta}^{D}\right)$, our considerations show that $\beta \mapsto \operatorname{det}\left(Q_{\alpha, \beta}^{D}\right)$ has $N^{\prime}$ 
real zeroes. Further, we have $\beta_{\left\lceil\left(N^{\prime}+1\right) / 2\right\rceil}(\alpha)<\alpha^{2}<\beta_{\left\lceil\left(N^{\prime}+3\right) / 2\right\rceil}(\alpha)$. This proves the proposition.

Proof of Theorem 1.5 Part (i) is proved by Proposition 5.6 whereas (ii) follows from Proposition 5.4 and Lemma 5.5 .

\section{A. Spectrum of the matrix $P^{D}$}

Here we compute for any integer $N^{\prime} \geq 3$ the eigenvalues of the $N^{\prime} \times N^{\prime}$-matrix $P^{D}$ appearing in the proof of Proposition 5.4.

Lemma A.1. If $N^{\prime}$ is even, the eigenvalues of $P^{D}$ are $4 N^{\prime}-3$ (with multiplicity one), 1 (with multiplicity $N^{\prime} / 2$ ), and -3 (with multiplicity $N^{\prime} / 2-1$ ). If $N^{\prime}$ is odd, the eigenvalues of $P^{D}$ are 1 (with multiplicity $\left(N^{\prime}-1\right) / 2$ ), -3 (with multiplicity $\left(N^{\prime}-3\right) / 2$ ), and $\frac{1}{2}\left(4 N^{\prime}-5\right)\left(1 \pm \sqrt{1+\frac{8\left(4 N^{\prime}-1\right)}{\left(4 N^{\prime}-5\right)^{2}}}\right)$ (each with multiplicity one).

Proof. Throughout this proof, $\operatorname{antidiag}\left(a_{1}, \ldots, a_{N^{\prime}}\right)$ denotes the "antidiagonal" $N^{\prime} \times N^{\prime}$ matrix $M$ with $M_{k l}=a_{l}$ if $k+l=N^{\prime}+1$ and $M_{k l}=0$ otherwise.

First consider the case where $N^{\prime}$ is even. We write $P^{D}$ in the form

$$
P^{D}=\operatorname{diag}(-1, \ldots,-1)+\operatorname{antidiag}(-2, \ldots,-2)+4 \cdot 1_{N^{\prime} \times N^{\prime}}
$$

and, with $\mu:=-1-\lambda$,

$$
P^{D}-\lambda \operatorname{Id}=\underbrace{\operatorname{diag}(\mu, \ldots, \mu)+\operatorname{antidiag}(-2, \ldots,-2)}_{=: L^{\left(N^{\prime}\right)}}+4 \cdot 1_{N^{\prime} \times N^{\prime}} .
$$

Here $1_{N^{\prime} \times N^{\prime}}$ denotes the $N^{\prime} \times N^{\prime}$-matrix whose entries are all equal to 1 .

We compute $\operatorname{det}\left(P^{D}-\lambda \mathrm{Id}\right)=\operatorname{det}\left(L^{\left(N^{\prime}\right)}+4 \cdot 1_{N^{\prime} \times N^{\prime}}\right)$ by column expansion. Note that in the column expansion of the determinant only those terms contribute which are determinants of matrices containing at most one column consisting of entries all equal to four. We obtain

$$
\operatorname{det}\left(P^{D}-\lambda \mathrm{Id}\right)=\operatorname{det}\left(L^{\left(N^{\prime}\right)}\right)+\sum_{j=1}^{N^{\prime}} \operatorname{det}\left(L_{j}^{\left(N^{\prime}\right)}\right)
$$

where $L_{j}^{\left(N^{\prime}\right)}$ is defined as the matrix $L^{\left(N^{\prime}\right)}$ with the $j$ th column replaced by the column $4 \cdot 1_{N^{\prime} \times 1}$. By expansion with respect to the first column and then the last column, the determinant of $L^{\left(N^{\prime}\right)}$ can be computed recursively,

$$
\operatorname{det}\left(L^{\left(N^{\prime}\right)}\right)=\left(\mu^{2}-2^{2}\right) \operatorname{det}\left(L^{\left(N^{\prime}-2\right)}\right) .
$$

Since $\operatorname{det}\left(L^{(2)}\right)=\mu^{2}-4$, it follows by induction that

$$
\operatorname{det}\left(L^{\left(N^{\prime}\right)}\right)=\left(\mu^{2}-4\right)^{N^{\prime} / 2} .
$$


To compute $\operatorname{det}\left(L_{1}^{\left(N^{\prime}\right)}\right)$, we expand the determinant in the same way and obtain the identity $\operatorname{det}\left(L_{1}^{\left(N^{\prime}\right)}\right)=4(\mu+2) \operatorname{det}\left(L^{\left(N^{\prime}-2\right)}\right)$, from which it follows that

$$
\operatorname{det}\left(L_{1}^{\left(N^{\prime}\right)}\right)=4(\mu+2)\left(\mu^{2}-4\right)^{N^{\prime} / 2-1} .
$$

Similarly one gets $\operatorname{det}\left(L_{2}^{\left(N^{\prime}\right)}\right)=\left(\mu^{2}-4\right) \operatorname{det}\left(L_{1}^{\left(N^{\prime}-2\right)}\right)$, and thus

$$
\operatorname{det}\left(L_{2}^{\left(N^{\prime}\right)}\right)=\operatorname{det}\left(L_{1}^{\left(N^{\prime}\right)}\right)=4(\mu+2)\left(\mu^{2}-4\right)^{N^{\prime} / 2-1} .
$$

For any $1<j<N^{\prime} / 2$, this procedure leads to $\operatorname{det}\left(L_{j}^{\left(N^{\prime}\right)}\right)=\left(\mu^{2}-4\right) \operatorname{det}\left(L_{j-1}^{\left(N^{\prime}-2\right)}\right)$ and hence

$$
\operatorname{det}\left(L_{j}^{\left(N^{\prime}\right)}\right)=\operatorname{det}\left(L_{1}^{\left(N^{\prime}\right)}\right)=4(\mu+2)\left(\mu^{2}-4\right)^{N^{\prime} / 2-1} .
$$

For $j>N^{\prime} / 2$, note that $\operatorname{det}\left(L_{j}^{\left(N^{\prime}\right)}\right)=\operatorname{det}\left(L_{N-j}^{\left(N^{\prime}\right)}\right)$, since $L_{j}^{\left(N^{\prime}\right)}$ and $L_{N-j}^{\left(N^{\prime}\right)}$ can be transformed into each other by exchanging the $j$ th and the $(N-j+1)$ th columns and then the $j$ th and the $(N-j+1)$ th rows. By $(81)-(84)$, we obtain

$$
\begin{aligned}
\operatorname{det}\left(P^{D}-\lambda \mathrm{Id}\right) & =\left(\mu^{2}-4\right)^{N^{\prime} / 2-1} \cdot\left(\left(\mu^{2}-4\right)+N^{\prime} \cdot 4(\mu+2)\right) \\
& =\left(\mu^{2}-4\right)^{N^{\prime} / 2-1}(\mu+2)\left(\mu-2+4 N^{\prime}\right) .
\end{aligned}
$$

Hence, if $N^{\prime}$ is even, the zeroes of $\operatorname{det}\left(P^{D}-\lambda\right.$ Id) are $\mu=2$ (with multiplicity $N^{\prime} / 2-1$ ), $\mu=-2$ (with multiplicity $N^{\prime} / 2$ ), and $\mu=-4 N^{\prime}+2$ (with multiplicity 1 ). Transforming back to $\lambda=-1-\mu$, we obtain the claimed eigenvalues.

It remains to consider the case where $N^{\prime}$ is odd. Again, we write

$P^{D}=\operatorname{diag}(-1, \ldots,-1, \overbrace{0}^{\left(\left(N^{\prime}+1\right) / 2\right)},-1, \ldots,-1)+\operatorname{antidiag}(-2, \ldots,-2)+4 \cdot 1_{N^{\prime} \times N^{\prime}}$.

With $\mu=-1-\lambda$ we get

$$
P^{D}-\lambda \mathrm{Id}=L^{\left(N^{\prime}\right)}+4 \cdot 1_{N^{\prime} \times N^{\prime}}
$$

with

$$
L^{\left(N^{\prime}\right)}=\left(\begin{array}{ccccc}
\mu & 0 & \ldots & 0 & -2 \\
0 & \ddots & & . & 0 \\
\vdots & & \mu-1 & & \vdots \\
0 & . \cdot & & \ddots & 0 \\
-2 & 0 & \ldots & 0 & \mu
\end{array}\right) .
$$

As above, we obtain the expansion 80 for the determinant of $P^{D}-\lambda$ Id. We expand $\operatorname{det}\left(L^{\left(N^{\prime}\right)}\right)$ with respect to the first column and then the last column, obtaining the recursion formula

$$
\operatorname{det}\left(L^{\left(N^{\prime}\right)}\right)=\left(\mu^{2}-4\right) \operatorname{det}\left(L^{\left(N^{\prime}-2\right)}\right),
$$


which together with $\operatorname{det}\left(L^{(1)}\right)=\mu-1$ leads to

$$
\operatorname{det}\left(L^{\left(N^{\prime}\right)}\right)=\left(\mu^{2}-4\right)^{\left(N^{\prime}-1\right) / 2}(\mu-1) .
$$

For $\operatorname{det}\left(L_{1}^{\left(N^{\prime}\right)}\right)$, we obtain the identity $\operatorname{det}\left(L_{1}^{\left(N^{\prime}\right)}\right)=4(\mu+2) \operatorname{det}\left(L^{\left(N^{\prime}-2\right)}\right)$ and hence

$$
\operatorname{det}\left(L_{1}^{\left(N^{\prime}\right)}\right)=4(\mu+2)\left(\mu^{2}-4\right)^{\left(N^{\prime}-3\right) / 2}(\mu-1) .
$$

More generally, for any $1<j<N^{\prime} / 2$, we have

$$
\operatorname{det}\left(L_{N-j}^{\left(N^{\prime}\right)}\right)=\operatorname{det}\left(L_{j}^{\left(N^{\prime}\right)}\right)=\operatorname{det}\left(L_{1}^{\left(N^{\prime}\right)}\right) .
$$

It remains to compute $\operatorname{det}\left(L_{\left(N^{\prime}+1\right) / 2}^{\left(N^{\prime}\right)}\right)$. Expanding $\operatorname{det}\left(L_{\left(N^{\prime}+1\right) / 2}^{\left(N^{\prime}\right)}\right)$ by the first column and then the last column, we obtain the recursion relation

$$
\operatorname{det}\left(L_{\left(N^{\prime}+1\right) / 2}^{\left(N^{\prime}\right)}\right)=\left(\mu^{2}-4\right) \operatorname{det}\left(L_{\left(\left(N^{\prime}-2\right)+1\right) / 2}^{\left(N^{\prime}-2\right)}\right)
$$

Together with $\operatorname{det}\left(L_{2}^{(3)}\right)=\operatorname{det}\left(\begin{array}{ccc}\mu & 4 & -2 \\ 0 & 4 & 0 \\ -2 & 4 & \mu\end{array}\right)=4\left(\mu^{2}-4\right)$, this implies

$$
\operatorname{det}\left(L_{\left(N^{\prime}+1\right) / 2}^{\left(N^{\prime}\right)}=4\left(\mu^{2}-4\right)^{\left(N^{\prime}-1\right) / 2} .\right.
$$

Hence, combining 85$]-88$, we obtain

$$
\begin{aligned}
\operatorname{det}\left(P^{D}-\lambda \mathrm{Id}\right)= & \left(\mu^{2}-4\right)^{\left(N^{\prime}-3\right) / 2} \\
& \cdot\left(\left(\mu^{2}-4\right)(\mu-1)+\left(N^{\prime}-1\right) \cdot 4(\mu+2)(\mu-1)+4\left(\mu^{2}-4\right)\right) \\
= & \left(\mu^{2}-4\right)^{\left(N^{\prime}-3\right) / 2}(\mu+2)\left(\mu^{2}+\left(4 N^{\prime}-3\right) \mu-\left(4 N^{\prime}+2\right)\right) .
\end{aligned}
$$

Hence, if $N^{\prime}$ is odd, the zeroes of $\operatorname{det}\left(P^{D}-\lambda\right.$ Id) are $\mu=2$ (with multiplicity $\left(N^{\prime}-3\right) / 2$ ), $\mu=-2$ (with multiplicity $\left(N^{\prime}-1\right) / 2$ ), and

$$
\mu=-\frac{1}{2}\left(4 N^{\prime}-3\right) \pm \frac{1}{2} \sqrt{16 N^{\prime 2}-8 N^{\prime}+17}
$$

(each with multiplicity 1 ). Transforming back to $\lambda=-1-\mu$, we obtain the claimed formulas for the eigenvalues in the case where $N^{\prime}$ is odd. This completes the proof of Lemma A.1.

Acknowledgments. It is a great pleasure to thank Bob Rink for valuable comments on earlier versions of this paper, and Gerda Schacher for her help with the graphics.

Research of A. Henrici was supported in part by the Swiss National Science Foundation. Research of T. Kappeler was supported in part by the Swiss National Science Foundation, the programme SPECT and the European Community through the FP6 Marie Curie RTN ENIGMA (MRTN-CT-2004-5652). 


\section{References}

[1] Bambusi, D., Ponno, A.: Korteweg-de Vries equation and energy sharing in Fermi-PastaUlam. Chaos 15, no. 1, 015107, 5 pp. (2005) Zbl 1080.37073 MR 2133458

[2] Bambusi, D., Ponno, A.: On metastability in FPU. Comm. Math. Phys. 264, 539-561 (2006) MR 2215616

[3] Berman, G. P., Izrailev, F. M.: The Fermi-Pasta-Ulam problem: fifty years of progress. Chaos 15, no. 1, 015104, 18 pp. (2005) Zbl 1080.37077 MR 2133455

[4] Cushman, R. H., Bates, L. M.: Global Aspects of Classical Integrable Systems. Birkhäuser, Basel (1997) Zbl 0882.58023 MR 1438060

[5] Fermi, E., Pasta, J., Ulam, S.: Studies of non linear problems. Los Alamos Rpt. LA-1940 (1955). In: Collected Papers of Enrico Fermi, Volume II, Univ. of Chicago Press, Chicago, 978-988 (1965); 2nd ed., Dekker, New York (2000)

[6] Henrici, A., Kappeler, T.: Birkhoff normal form for the periodic Toda lattice. In: Integrable Systems and Random Matrices, Contemp. Math. 458, Amer. Math. Soc., 11-29 (2008) Zbl 1149.37031 MR 2411898

[7] Henrici, A., Kappeler, T.: Results on normal forms for FPU chains. Comm. Math. Phys. 278, 145-177 (2008) Zbl pre05306078 MR 2367802

[8] Nishida, T.: A note on an existence of conditionally periodic oscillation in a one-dimensional lattice. Mem. Fac. Engrg. Kyoto Univ. 33, 27-34 (1971) MR 0279387

[9] Pöschel, J.: Integrability of Hamiltonian systems on Cantor sets. Comm. Pure Appl. Math. 35, 653-695 (1982) Zbl 0542.58015 MR 0668410

[10] Pöschel, J.: On Nekhoroshev's estimate at an elliptic equilibrium. Int. Math. Res. Notices 4, 203-215 (1999) Zbl 0918.58026 MR 1677275

[11] Rink, B.: Symmetry and resonance in periodic FPU chains. Comm. Math. Phys. 218, 665-685 (2001) Zbl 0997.37057 MR 1831098

[12] Rink, B.: Direction reversing travelling waves in the Fermi-Pasta-Ulam chain. J. Nonlinear Sci. 12, 479-504 (2002) Zbl 1012.37051 MR 1923389

[13] Rink, B.: Proof of Nishida's conjecture on anharmonic lattices. Comm. Math. Phys. 261, 613627 (2006) Zbl 1113.82045 MR 2197541

[14] Toda, M.: Theory of Nonlinear Lattices. 2nd English ed., Springer Ser. Solid-State Sci. 20, Springer, Berlin (1989) Zbl 0694.70001 MR 0971987.

[15] Weissert, T. P.: The Genesis of Simulation in Dynamics: Pursuing the Fermi-Pasta-Ulam Problem. Springer, New York (1997) Zbl 0908.58068 MR 1477158 